NBER WORKING PAPER SERIES

\title{
ESTIMATING THE EFFECT OF TREATMENTS ALLOCATED BY RANDOMIZED WAITING LISTS.
}

Clément de Chaisemartin

Luc Behaghel

Working Paper 26282

http://www.nber.org/papers/w26282

\author{
NATIONAL BUREAU OF ECONOMIC RESEARCH \\ 1050 Massachusetts Avenue \\ Cambridge, MA 02138 \\ September 2019
}

We would like to thank Hugo Botton for outstanding research assistance. We are very grateful to Chris Blattman and Jeannie Annan for making their data publicly available, and for answering all our questions. We are also very grateful to Josh Angrist, Bart Cockx, Xavier D'Haultfoeuille, Thomas Le Barbanchon, Chang Lee, Ulrich Mueller, Heather Royer, Doug Steigerwald, Chris Walters, three anonymous referees, members of the UCSB econometrics research group, and seminar participants at: the 2017 California Econometrics conference, the 2017 Labor and Education workshop of the NBER Summer Institute, Louvain-la-Neuve, the Paris School of Economics, UC Santa Barbara, UC San Diego, and Warwick for their helpful comments. The views expressed herein are those of the authors and do not necessarily reflect the views of the National Bureau of Economic Research.

NBER working papers are circulated for discussion and comment purposes. They have not been peer-reviewed or been subject to the review by the NBER Board of Directors that accompanies official NBER publications.

(C) 2019 by Clément de Chaisemartin and Luc Behaghel. All rights reserved. Short sections of text, not to exceed two paragraphs, may be quoted without explicit permission provided that full credit, including $\odot$ notice, is given to the source. 
Estimating the Effect of Treatments Allocated by Randomized Waiting Lists.

Clément de Chaisemartin and Luc Behaghel

NBER Working Paper No. 26282

September 2019

JEL No. C21,C26

\begin{abstract}
$\underline{\text { ABSTRACT }}$
Oversubscribed treatments are often allocated using randomized waiting lists. Applicants are ranked randomly, and treatment offers are made following that ranking until all seats are filled. To estimate causal effects, researchers often compare applicants getting and not getting an offer. We show that those two groups are not statistically comparable. Therefore, the estimator arising from that comparison is inconsistent when the number of waitlists goes to infinity. We propose a new estimator, and show that it is consistent, provided the waitlists have at least two seats. Finally, we revisit an application, and we show that using our estimator can lead to significantly different results from those obtained using the commonly used estimator.
\end{abstract}

Clément de Chaisemartin

Department of Economics

University of California at Santa Barbara

Santa Barbara, CA 93106

and NBER

clementdechaisemartin@ucsb.edu

Luc Behaghel

Paris School of Economics - Inra

48 boulevard Jourdan

75014 PARIS

FRANCE

luc.behaghel@ens.fr 


\section{Introduction}

Often times, some individuals who apply for a treatment are non-takers. They decline to get treated when they receive an offer, for instance because they then realize that their benefit from treatment is lower than they thought. When a treatment is oversubscribed but some applicants are non-takers, an appealing way of allocating the available seats is to use randomized waitlists. First, applicants are ranked randomly. Then, if $S$ seats are available, an initial round of offers takes place, whereby the first $S$ applicants get an offer. If $r$ of them decline it, a subsequent round of offers takes place whereby the next $r$ applicants get an offer. Offers stop when all the seats have been filled. This allocation method is fair: each taker has the same probability of being treated; it is also efficient: no seat for treatment remains unused, despite the presence of non-takers. Therefore, oversubscribed treatments with non-takers are often allocated by randomized waitlists. We conducted a survey, and found 43 articles studying treatments allocated by randomized waitlists, ranging from charter schools in the USA to agricultural trainings in Liberia. These treatments often have capacity constraints for various groups of applicants. For instance, a charter school may have 20 seats available in 7 th grade and 25 seats in 8 th grade. Then, a lottery takes place in each group.

As applicants are ranked randomly, it may be possible to form two comparable groups with different likelihoods of getting an offer. One could then compare those two groups to estimate the effect of the treatment. In practice, researchers have used two types of comparisons. Some researchers have compared applicants getting and not getting an initial offer, thus giving rise to the so-called initial-offer (IO) estimators. Other researchers have compared applicants ever and never getting an offer, thus giving rise to the so-called ever-offer (EO) estimators. When several lotteries were conducted, as in the charter school example above, researchers have often included waitlist fixed effects in their specifications, to ensure they compare applicants within and not across waitlists. In our survey, 22 articles used the EO estimator, 20 used the IO estimator, and a handful used other estimators. Overall, practices are not standardized.

We start by showing that the expected proportion of takers is strictly greater among applicants ever getting an offer than among applicants never getting one. Intuitively, this is because offers continue until all seats are filled, so the last applicant getting an offer must by construction be a taker. Moreover, when waitlist fixed effects are included in the estimation, they induce an endogenous reweighting of waitlists that usually further increases this imbalance between the two groups, as we explain in more detail in Section 2.

Then, we show that due to this imbalance, the EO estimator is inconsistent when the number of waitlists goes to infinity. In our survey, the median of the number of waitlists divided by the number of applicants per waitlist is 1.9 , and $25 \%$ of the articles have more than 100 waitlists and less than 40 applicants per waitlist. This motivates the asymptotic sequence we consider. By contrast, if the number of applicants and takers per waitlist goes to infinity, the asymptotic 
bias of the EO estimator goes to 0 .

We show that dropping the last applicant getting an offer in each waitlist is sufficient to restore the comparability between applicants getting and not getting an offer. Based on this result, we propose a new estimator of the treatment effect. It is built out of comparisons of applicants that get and do not get an offer in each waitlist, downweighting applicants that accept their offer by an amount equivalent to dropping one of them. Then, our estimator takes a weighted average of those within-waitlist comparisons, with a weighting scheme that avoids the endogeneous reweighting induced by the waitlist fixed effects. We refer to that estimator as the doubly-reweighted ever-offer estimator (DREO). We show that our estimator is consistent and asymptotically normal when the number of waitlists goes to infinity.

Contrary to subsequent-round offers, initial offers are only a function of applicants' random ranks in the waitlist. Therefore, applicants getting and not getting an initial offer are statistically comparable, and the IO estimator is also consistent. However, we show that the asymptotic variance of that estimator is often much larger than that of the DREO estimator, so using it will often result in large efficiency losses.

We use our results to revisit Blattman \& Annan (2016), who studied the effects of an agricultural training. The DREO estimator is significantly different from the EO estimator computed by the authors for some of the outcomes they considered. ${ }^{1}$

The remainder of the paper is organized as follows. Section 2 uses a simple example to give the intuition of our results. Section 3 presents our main results. Section 4 presents our empirical application. Appendix A presents the proofs. In our Web appendix, we present our survey of articles that have used randomized waitlists, we show that some of the assumptions adopted in the paper can be relaxed, we present some simulations, and we revisit another application.

\section{Introducing the results through a simple example}

We start with a simple example. We consider a waitlist where five applicants compete for three seats. Four applicants are takers $(T)$ and one is a non-taker $(N T)$, meaning that she will refuse to get treated if she gets an offer. Applicants are randomly ranked, and treatment offers are made following that ranking until all seats are filled. There are 5! possible orderings of the applicants, that can be divided into 5 groups of 4 ! orderings, according to the rank of the non-taker. Table 1 displays those five groups of orderings, hereafter referred to, slightly abusively, as "orderings". For each ordering, applicants getting an offer are depicted in italics, while those not getting an offer are depicted in bold. In ordering 1 and 2, the first three applicants are takers, so offers stop after the third offer. In orderings 3,4 , and 5 , one of the first three applicants is a non-taker, so a fourth offer is made; then the next applicant is a taker so offers stop as the available seats have been filled.

\footnotetext{
${ }^{1}$ A Stata adofile computing the DREO estimator is available from the authors' website.
} 
The first issue with the EO estimator is that, on average, applicants getting an offer bear a higher proportion of takers than applicants not getting an offer. Each ordering has a 0.20 probability of being selected. Across the five orderings, the expected share of takers among applicants getting an offer is $0.2 \times(1+1+3 / 4+3 / 4+3 / 4)=17 / 20$. On the other hand, the expected share of takers among applicants not getting an offer is $0.2 \times(1 / 2+1 / 2+1+1+1)=$ $4 / 5$. Intuitively, this imbalance arises because offers stop when sufficiently many takers have accepted an offer. This endogenous stopping rule creates a positive correlation between getting an offer and being a taker. When the average potential outcomes of takers and non takers differ, ${ }^{2}$ this imbalance implies that applicants getting and not getting an offer are not statistically comparable: those two groups have different average potential outcomes.

Table 1: Applicants getting and not getting an offer in an example

\begin{tabular}{c|c|c|c|c} 
Ordering 1 & Ordering 2 & Ordering 3 & Ordering 4 & Ordering 5 \\
$T$ & $T$ & $T$ & $T$ & $N T$ \\
$T$ & $T$ & $T$ & $N T$ & $T$ \\
$T$ & $T$ & $N T$ & $T$ & $T$ \\
$\mathbf{T}$ & $\mathbf{N T}$ & $T$ & $T$ & $T$ \\
NT & $\mathbf{T}$ & $\mathbf{T}$ & $\mathbf{T}$ & $\mathbf{T}$
\end{tabular}

The second issue with the EO estimator arises from the inclusion of fixed effects when pooling waitlists. Assume that one pools waitlists that all have four takers, one non-taker, and three seats. In some waitlists, the realized ordering of takers and non-takers is Ordering 1 in Table 1 , in other waitlists the realized ordering is Ordering 2 , etc. With several waitlists, it follows from, e.g., Equation (3.3.7) in Angrist \& Pischke (2008), that the EO estimator with waitlist fixed effects is a weighted average of the EO estimators in each waitlist, that gives more weight to waitlists where the share of applicants getting an offer is closer to $1 / 2$. In our example, $3 / 5$ of applicants get an offer in waitlists with Ordering 1 or 2 , while $4 / 5$ of applicants get an offer in waitlists with Ordering 3, 4, or 5. Accordingly, waitlists with Ordering 1 or 2 receive more weight. But those are precisely the waitlists where the proportion of takers among applicants getting an offer is the highest. Therefore, the reweighting of waitlists induced by the fixed effects aggravates the over-representation of takers among applicants getting an offer.

The DREO estimator we propose addresses those two issues. Firstly, in our example dropping the last taker getting an offer is sufficient to solve the endogenous stopping rule issue. Table 2 shows that then, the expected share of takers among applicants getting an offer is equal to $0.2 \times(1+1+2 / 3+2 / 3+2 / 3)=4 / 5$, the same as among applicants not getting an offer. Still, dropping the last taker getting an offer is arbitrary: dropping the first or the second

\footnotetext{
${ }^{2}$ This is often the case. Abadie et al. (2002) and Crépon et al. (2015) are just a few examples of the many papers that have found large differences between the average potential outcomes of takers and non-takers.
} 
would have the same effect. Besides, doing so reduces the sample size and statistical precision. Instead, one can give to the three of them a weight equal to $2 / 3$ : this reduces the expected share of takers among applicants getting an offer by the same amount as dropping one. Secondly, instead of using fixed effects to pool waitlists, we simply take an average of the estimators in each waitlist, weighting waitlists proportionally to their number of applicants. These weights are independent of how many offers one has to make to fill the available seats, which solves the second issue of the EO estimator. Table 2 shows that this second reweighting is necessary. Even after downweighting takers getting an offer, including waitlist fixed effects would still lead to over-represent takers among applicants getting an offer. Indeed, doing so gives more weight to waitlists with ordering 1 or 2 , where $1 / 2$ of applicants get an offer, while those are the waitlists where the proportion of takers among applicants getting an offer is the highest.

Table 2: Applicants getting and not getting an offer, dropping the last taker getting an offer

\begin{tabular}{c|c|c|c|c} 
Ordering 1 & Ordering 2 & Ordering 3 & Ordering 4 & Ordering 5 \\
$T$ & $T$ & $T$ & $T$ & $N T$ \\
$T$ & $T$ & $T$ & $N T$ & $T$ \\
& & $N T$ & $T$ & $T$ \\
T & NT & & & \\
NT & $\mathbf{T}$ & $\mathbf{T}$ & $\mathbf{T}$ & $\mathbf{T}$
\end{tabular}

\section{Main results}

\subsection{Assumptions and parameter of interest}

Throughout the paper, we consider the following set-up.

Assumption 1 (Set-up)

a) Applicants for a binary treatment are divided into $K$ mutually exclusive waitlists. For every $k \in\{1 . . K\}, N_{k}$ denotes the number of applicants in waitlist $k . N_{k}$ is non stochastic.

b) In each waitlist, $S_{k}$ seats are available, and are allocated as follows: applicants are ranked, and treatment offers are made following that order until $S_{k}$ applicants have accepted to get treated or all applicants have received an offer. $S_{k}$ is non stochastic.

c) Applicants that do not get an offer cannot get treated.

In Section 4 in the Web appendix, we consider various extensions of this set-up. For instance, we show that our results remain unchanged if we allow for the possibility that some applicants manage to get treated even if they do not receive an offer. Similarly, we allow for the possibility 
that some applicants may participate in several waiting-lists, or that the treatment may not be binary. But for now we focus on the basic set-up outlined in Assumption 1.

Then, we assume that ranks are randomly assigned to applicants. Let $R_{i k}$ denote the rank assigned to applicant $i$ in waitlist $k$, let $L_{k}$ denote the number of applicants getting an offer in waitlist $k$, and let $Z_{i k}=1\left\{R_{i k} \leq L_{k}\right\}$ denote whether applicant $i$ gets an offer, the so-called ever-offer instrument. Let $D_{i k}(1)$ denote her potential treatment if she gets an offer, and let $D_{i k}$ denote her observed treatment. Under point c) of Assumption 1, $D_{i k}=Z_{i k} D_{i k}(1)$. For every $d \in\{0,1\}$, let $Y_{i k}(d)$ denote her potential outcome if $D_{i k}=d,{ }^{3}$ and let $Y_{i k}=Y_{i k}\left(D_{i k}\right)$ denote her observed outcome. Let

$$
\mathcal{P}_{k}=\left(\left(D_{1 k}(1), Y_{1 k}(0), Y_{1 k}(1)\right), \ldots,\left(D_{N_{k} k}(1), Y_{N_{k} k}(0), Y_{N_{k} k}(1)\right)\right)
$$

be a vector stacking the potential treatments and outcomes of the applicants in waitlist $k$. For any integer $j$, let $\Pi_{j}$ denote the set of permutations of $\{1 . . j\}$. Let $\mathcal{R}_{k}=\left(R_{1 k}, \ldots, R_{N_{k} k}\right)$ denote the ranks assigned to applicants 1 to $N_{k}$ in waitlist $k$.

Assumption 2 (Randomly assigned ranks)

For all $k \in\{1 . . K\}$ and $\left(r_{1}, \ldots, r_{N_{k}}\right) \in \Pi_{N_{k}}, P\left(\mathcal{R}_{k}=\left(r_{1}, \ldots, r_{N_{k}}\right) \mid \mathcal{P}_{k}\right)=\frac{1}{N_{k} !}$.

Assumption 2 requires that the ranks assigned to applicants be independent of their potential treatments and outcomes, and uniformly distributed on $\Pi_{N_{k}}$. It implies that each applicant has the same probability of being in the first, second, ..., or last rank.

Finally, we consider a last assumption. Let applicants with $D_{i k}(1)=1\left(\right.$ resp. $\left.D_{i k}(1)=0\right)$ be referred to as takers (resp. non-takers). For every $k \in\{1 . . K\}$, let $T_{k}=\sum_{i=1}^{N_{k}} D_{i k}(1)$ denote the number of takers in waitlist $k$.

Assumption 3 (Strictly more takers than seats)

For every $k \in\{1 . . K\}, 2 \leq S_{k}<T_{k}$.

Assumption 3 requires that each waitlist have at least two seats, so waitlists with less than two seats have to be dropped from the analysis. Assumption 3 also requires that each waitlist have strictly more takers than seats. When all the seats available in a waitlist get filled, it must be that $S_{k} \leq T_{k}$, but it is still possible that $S_{k}=T_{k}$ : all applicants not getting an offer might be non-takers. Still, in Section 4.1 of the Web appendix we propose a statistical test of whether $S_{k}<T_{k}$ for all $k$, or $S_{k}=T_{k}$ for some $k$.

Let $T=\sum_{k=1}^{K} T_{k}$ denote the total number of takers. Our parameter of interest is

$$
\Delta_{K}=E\left(\frac{1}{T} \sum_{(i, k): D_{i k}(1)=1}\left[Y_{i k}(1)-Y_{i k}(0)\right]\right),
$$

the local average treatment effect of the takers.

\footnotetext{
${ }^{3}$ We implicitly assume that getting an offer does not have a direct effect on the outcome, the so-called exclusion restriction, see Angrist et al. (1996).
} 


\subsection{The Doubly Reweighted Ever Offer estimator}

Let $N=\sum_{k=1}^{K} N_{k}$ and $\bar{N}=\frac{N}{K}$ respectively denote the total number of applicants and the average number of applicants per waitlist. Let $\mathcal{I}=\left\{(i, k) \in \mathbb{N}^{2}: i \in\left\{1 . . N_{k}\right\}, k \in\{1 . . K\}\right\}$, and for every $(i, k) \in \mathcal{I}$, let $w_{i k}=1-\frac{Z_{i k} D_{i k}}{S_{k}} \cdot w_{i k}$ is equal to $1-\frac{1}{S_{k}}$ for applicants that get and accept an offer, and to 1 for everyone else. As $S_{k}$ takers receive an offer in each waitlist, weighting applicants getting an offer by $w_{i k}$ decreases the share of takers among them by the same amount as dropping one taker, as illustrated in the numerical example in Section 2.

The DREO estimator of $\Delta_{K}$ is defined as

$$
\widehat{\Delta}=\frac{\frac{1}{K} \sum_{k=1}^{K} \frac{N_{k}}{\bar{N}}\left(\frac{1}{L_{k}-1} \sum_{i: Z_{i k}=1} w_{i k} Y_{i k}-\frac{1}{N_{k}-L_{k}} \sum_{i: Z_{i k}=0} Y_{i k}\right)}{\frac{1}{K} \sum_{k=1}^{K} \frac{N_{k}}{N} \frac{1}{L_{k}-1} \sum_{i: Z_{i k}=1} w_{i k} D_{i k}} .
$$

$\widehat{\Delta}$ can be computed through a 2 SLS regression. Let $L=\sum_{k=1}^{K} L_{k}$, and let

$$
w_{i k}^{D R}=w_{i k}\left(Z_{i k} \times \frac{L-K}{N-K} \times \frac{N_{k}}{L_{k}-1}+\left(1-Z_{i k}\right) \times \frac{N-L}{N-K} \times \frac{N_{k}}{N_{k}-L_{k}}\right)
$$

be a weighting scheme combining $w_{i k}$ with propensity score reweighting. One can show that $\widehat{\Delta}$ is equal to the coefficient of $D_{i k}$ in a 2SLS regression of $Y_{i k}$ on $D_{i k}$ using $Z_{i k}$ as the instrument, and weighted by $w_{i k}^{D R}$. Importantly, note that under Assumption $1, S_{k}=\sum_{i=1}^{N_{k}} Z_{i k} D_{i k}$, so observing $\left(Z_{i k}, D_{i k}, Y_{i k}\right)_{(i, k) \in\left\{1 . . N_{k}\right\} \times\{1 . . K\}}$ is sufficient to compute $\widehat{\Delta}$.

Our main result relies on the following lemma:

Lemma 3.1 If Assumptions 1-3 hold, then for all $k \in\{1 . . K\}$,

a) $E\left(\frac{1}{K} \sum_{k=1}^{K} \frac{N_{k}}{N}\left(\frac{1}{L_{k}-1} \sum_{i: Z_{i k}=1} w_{i k} Y_{i k}-\frac{1}{N_{k}-L_{k}} \sum_{i: Z_{i k}=0} Y_{i k}\right)\right)=E\left(\frac{1}{N} \sum_{(i, k) \in \mathcal{I}}\left[Y_{i k}\left(D_{i k}(1)\right)-Y_{i k}(0)\right]\right)$,

b) $E\left(\frac{1}{K} \sum_{k=1}^{K} \frac{N_{k}}{\bar{N}} \frac{1}{L_{k}-1} \sum_{i: Z_{i k}=1} w_{i k} D_{i k}\right)=E\left(\frac{1}{N} \sum_{(i, k) \in \mathcal{I}} D_{i k}(1)\right)$.

The intuition of point a) of the theorem goes as follows. As the numerical example in Section 2 illustrates, one can show that in each waitlist, $w_{i k}$-reweighted applicants getting an offer are statistically comparable to applicants not getting an offer. Therefore, the only difference between these two groups is that one receives an offer and not the other one. Accordingly, $\frac{1}{L_{k}-1} \sum_{i: Z_{i k}=1} w_{i k} Y_{i k}-\frac{1}{N_{k}-L_{k}} \sum_{i: Z_{i k}=0} Y_{i k}$, the difference between the average outcome of the two groups, is an unbiased estimator of $E\left(\frac{1}{N_{k}} \sum_{i=1}^{N_{k}}\left[Y_{i k}\left(D_{i k}(1)\right)-Y_{i k}(0)\right]\right)$, the intention to treat (ITT) effect of getting an offer on applicants' outcome in waitlist $k$. The numerator of $\widehat{\Delta}$ is an average of those unbiased within-waitlist comparisons, that gives to each waitlist a weight proportional to its number of applicants. Therefore, this numerator is an unbiased estimator of $E\left(\frac{1}{N} \sum_{(i, k) \in \mathcal{I}}\left[Y_{i k}\left(D_{i k}(1)\right)-Y_{i k}(0)\right]\right)$, the intention to treat effect among all applicants. The intuition of point $b$ ) is similar. 
We now derive the asymptotic distribution of $\widehat{\Delta}$. In our survey of articles that have used randomized waitlists, the median number of waitlists used in the analysis is equal to 64 . Therefore, we consider a sequence where $K$, the number of waitlists, goes to infinity. An alternative would be to consider a sequence where the number of applicants per waitlist goes to infinity, but in our survey the median of waitlists divided by applicants per waitlist is equal to 1.9, so the former asymptotic may be more appropriate in a majority of applications. For all $k \in\{1 . . K\}$, let $R F_{k}=\frac{N_{k}}{\bar{N}}\left[\frac{1}{L_{k}-1} \sum_{i: Z_{i k}=1} w_{i k} Y_{i k}-\frac{1}{N_{k}-L_{k}} \sum_{i: Z_{i k}=0} Y_{i k}\right]$ and $F S_{k}=$ $\frac{N_{k}}{\bar{N}} \frac{1}{L_{k}-1} \sum_{i: Z_{i k}=1} w_{i k} D_{i k}$. Let also $F S=\lim _{K \rightarrow+\infty} \frac{1}{K} \sum_{k=1}^{K} E\left(F S_{k}\right)$ and $\Delta=\lim _{K \rightarrow+\infty} \Delta_{K}$, where Assumption 4 below ensures that those limits exist. Finally, for all $k$ let $\Lambda_{k}=\frac{R F_{k}-\Delta F S_{k}}{F S}$.

Assumption 4 (Technical assumptions to derive the asymptotic distribution of $\widehat{\Delta}$ )

a) The vectors $\left(\mathcal{P}_{k}, \mathcal{R}_{k}\right)_{1 \leq k \leq K}$ are mutually independent.

b) For all $(i, k) \in \mathcal{I}, D_{i k}(1)$ is non-stochastic.

c) For every $k, N_{k} \leq N^{+}$, for some integer $N^{+}$.

d) $\sup _{k \in \mathbb{N} *} E\left(\left|R F_{k}\right|^{2+\delta}\right)<+\infty$, for some strictly positive $\delta$.

e) The following sequences have finite limits when $K \rightarrow+\infty$ : i) $\frac{1}{K} \sum_{k=1}^{K} E\left(R F_{k}\right)$, ii) $\frac{1}{K} \sum_{k=1}^{K} E\left(F S_{k}\right)$, iii) $\frac{1}{K} \sum_{k=1}^{K} V\left(R F_{k}\right)$, iv) $\left.\frac{1}{K} \sum_{k=1}^{K} V\left(F S_{k}\right), v\right) \frac{1}{K} \sum_{k=1}^{K} E\left(R F_{k} F S_{k}\right)$, vi) $\frac{1}{K} \sum_{k=1}^{K} E\left(\left|R F_{k}-E\left(R F_{k}\right)\right|^{2+\delta}\right)$, vii) $\frac{1}{K} \sum_{k=1}^{K} E\left(\left|F S_{k}-E\left(F S_{k}\right)\right|^{2+\delta}\right)$, and viii) $\frac{1}{K} \sum_{k=1}^{K} E\left(\left|\Lambda_{k}-E\left(\Lambda_{k}\right)\right|^{2+\delta}\right)$.

Typically, the lotteries determining applicants' ranks are independent across waitlists, so by design the vectors $\left(\mathcal{R}_{k}\right)_{1 \leq k \leq K}$ are independent, and $\left(\mathcal{R}_{k}\right)_{1 \leq k \leq K}$ is independent of $\left(\mathcal{P}_{k}\right)_{1 \leq k \leq K}$. Then, point a) of Assumption 4 only requires that the vectors $\left(\mathcal{P}_{k}\right)_{1 \leq k \leq K}$ be independent. This is often plausible, for instance when the waitlists correspond to different schools.

Point b) requires that whether an applicant is a taker or a non-taker be non-stochastic, a commonly-made assumption in the randomization-inference literature with imperfect compliance (see, e.g., Imbens \& Rosenbaum, 2005). Under this assumption, the denominator of $\Delta_{K}$ is non-stochastic, so it follows from Lemma 3.1 that $\Delta_{K}=\frac{1 / K \sum_{k=1}^{K} E\left(R F_{k}\right)}{1 / K \sum_{k=1}^{K} E\left(F S_{k}\right)}$. Then, the asymptotic normality of $\sqrt{K}\left(\widehat{\Delta}-\Delta_{K}\right)$ follows from the central limit theorem and from the linearization of a ratio. Theorem 3.1 still holds without point b), provided the target parameter $\Delta_{K}$ is replaced by $\tilde{\Delta}_{K}=E\left(\sum_{k=1}^{K} \frac{T_{k}}{\sum_{k=1}^{K} E\left(T_{k}\right)} L A T E_{k}\right)$, where $L A T E_{k}=$ $\frac{1}{T_{k}} \sum_{i: D_{i k}(1)=1}\left[Y_{i k}(1)-Y_{i k}(0)\right]$ is the LATE in waitlist $k$. Indeed, it follows from Lemma 3.1 that $\tilde{\Delta}_{K}=\frac{1 / K \sum_{k=1}^{K} E\left(R F_{k}\right)}{1 / K \sum_{k=1}^{K} E\left(F S_{k}\right)}$ even if point b) fails. Overall, point b) of Assumption 4 is not crucially important for Theorem 3.1 to hold, but it simplifies the target parameter. ${ }^{4}$

Point c) requires that the number of applicants per waitlist be uniformly bounded by some constant $N^{+}$. Points d) and e) are technical conditions ensuring we can apply Liapunov's

\footnotetext{
${ }^{4}$ Theorem 3.1 also holds (with $\Delta$, the limit of $\Delta_{K}$, as the target parameter) if point b) is replaced by the
} 
central limit theorem to $\left(R F_{k}\right)_{k \in \mathbb{N}^{*}},\left(F S_{k}\right)_{k \in \mathbb{N}^{*}}$, and $\left(\Lambda_{k}\right)_{k \in \mathbb{N}^{*}}$, and the weak law of large numbers in Gut (1992) to $\left(R F_{k}\right)_{k \in \mathbb{N}^{*}},\left(F S_{k}\right)_{k \in \mathbb{N}^{*}},\left(R F_{k}^{2}\right)_{k \in \mathbb{N}^{*}}$, and $\left(F S_{k}^{2}\right)_{k \in \mathbb{N}^{*}}$ d d) for instance holds if the potential outcomes $Y_{i k}(0)$ and $Y_{i k}(1)$ have a bounded support.

Let $\sigma^{2}=\lim _{K \rightarrow+\infty} \frac{1}{K} \sum_{k=1}^{K} V\left(\Lambda_{k}\right), \sigma_{+}^{2}=\lim _{K \rightarrow+\infty}\left[\frac{1}{K} \sum_{k=1}^{K} E\left(\Lambda_{k}^{2}\right)-\left(\frac{1}{K} \sum_{k=1}^{K} E\left(\Lambda_{k}\right)\right)^{2}\right]$, $\widehat{\Lambda}_{k}=\frac{R F_{k}-\widehat{\Delta} F S_{k}}{\frac{1}{K} \sum_{k=1}^{K} F S_{k}}$, and $\widehat{\sigma}_{+}^{2}=\frac{1}{K} \sum_{k=1}^{K}\left(\widehat{\Lambda}_{k}-\frac{1}{K} \sum_{j=1}^{K} \widehat{\Lambda}_{j}\right)^{2}$.

Theorem 3.1 If Assumptions 1-4 hold, $\sqrt{K}\left(\widehat{\Delta}-\Delta_{K}\right) \stackrel{d}{\longrightarrow} \mathcal{N}\left(0, \sigma^{2}\right)$ and $\widehat{\sigma}_{+}^{2} \stackrel{p}{\longrightarrow} \sigma_{+}^{2} \geq \sigma^{2}$.

Theorem 3.1 implies that $\widehat{\Delta}$ is an asymptotically normal estimator of $\Delta_{K}$ when the number of waitlists goes to infinity. As is usually the case for estimators constructed using independent but not identically distributed random variables (see e.g. Liu \& Singh, 1995), the asymptotic variance $\sigma^{2}$ of $\widehat{\Delta}$ can only be conservatively estimated: we provide a consistent estimator of $\sigma_{+}^{2}$, an upper bound of $\sigma^{2}$. That estimator can then be used to build conservative confidence intervals for $\Delta_{K} .{ }^{5}$ When all the $\Lambda_{k}$ have the same expectation, something that for instance happens when all waitlists have the same number of applicants, the same expectation of the proportion of takers, and the same expectations of takers' and non takers' potential outcomes, $\sigma_{+}^{2}=\sigma^{2}$ so those confidence intervals are exact. Finally, in simulations shown in Section 3.4 of the Web appendix, we find that the asymptotic distribution in Theorem 3.1 approximates the distribution of $\widehat{\Delta}$ well if 20 waitlists or more are used in the analysis. This suggests that articles using more than 20 waitlists may rely on Theorem 3.1 for inference, while articles using less than 20 waitlists may not.

\subsection{Comparison with the Ever Offer and Initial Offer estimators}

\subsubsection{Comparison with the Ever Offer estimator}

Let $\widehat{\beta}_{F E}^{E}$ be the coefficient of $D_{i k}$ in a 2SLS regression of $Y_{i k}$ on $D_{i k}$ and waitlist fixed effects, using $Z_{i k}$ as the instrument for $D_{i k}$. We refer to $\widehat{\beta}_{F E}^{E}$ as the EO estimator. The derivation of its limit relies on Assumption 6, another technical assumption, that is stated in the proofs.

following conditions:

$$
\begin{aligned}
& \sqrt{K}\left(\frac{1}{K} \sum_{k=1}^{K} E\left(R F_{k}\right)-\lim _{K \rightarrow+\infty} \frac{1}{K} \sum_{k=1}^{K} E\left(R F_{k}\right)\right) \underset{K \rightarrow+\infty}{\longrightarrow} 0 \\
& \sqrt{K}\left(\frac{1}{K} \sum_{k=1}^{K} E\left(F S_{k}\right)-\lim _{K \rightarrow+\infty} \frac{1}{K} \sum_{k=1}^{K} E\left(F S_{k}\right)\right) \underset{K \rightarrow+\infty}{\longrightarrow} 0 .
\end{aligned}
$$

These two conditions automatically hold if $E\left(R F_{k}\right)$ and $E\left(F S_{k}\right)$ are the same in every waitlist. But they can also hold with heterogeneous waitlists. For instance, they hold if the sequences $\left(E\left(R F_{k}\right)\right)_{k \in \mathbb{N}^{*}}$ and $E\left(F S_{k}\right)_{k \in \mathbb{N}^{*}}$ are periodic, which corresponds to an asymptotic where the sample grows by duplicating the original sample.

${ }^{5}$ Conservative variance estimators also arise in other articles studying treatment effect estimation in randomized experiments (see e.g. Neyman, 1923). 
Assumption 6 ensures that the limits in the definition of $w_{k}$ and $B$ below exist. Let

$$
\begin{aligned}
& w_{k}= \frac{\frac{S_{k}\left(N_{k}-S_{k} \frac{N_{k}+1}{T_{k}+1}\right)}{N_{k}}}{\lim _{K \rightarrow+\infty} \frac{1}{K} \sum_{j=1}^{K} E\left(\frac{S_{j}\left(N_{j}-S_{j} \frac{N_{j}+1}{T_{j}+1}\right)}{N_{j}}\right)}, \\
& B=\frac{\lim _{K \rightarrow+\infty} \frac{1}{K} \sum_{k=1}^{K} E\left(\frac{S_{k}\left(N_{k}-T_{k} \frac{N_{k}+1}{T_{k}+1}\right)}{N_{k}}\left[\frac{1}{T_{k}} \sum_{i: D_{i k}(1)=1} Y_{i k}(0)-\frac{1}{N_{k}-T_{k}} \sum_{i: D_{i k}(1)=0} Y_{i k}(0)\right]\right)}{\lim _{K \rightarrow+\infty} \frac{1}{K} \sum_{k=1}^{K} E\left(\frac{S_{k}\left(N_{k}-S_{k} \frac{N_{k}+1}{T_{k}+1}\right)}{N_{k}}\right)} .
\end{aligned}
$$

Theorem 3.2 If Assumptions 1 -4 and 6 hold,

$$
\widehat{\beta}_{F E}^{E} \stackrel{p}{\longrightarrow} \lim _{K \rightarrow+\infty} \frac{1}{K} \sum_{k=1}^{K} E\left(w_{k} \frac{1}{T_{k}} \sum_{i: D_{i k}(1)=1}\left[Y_{i k}(1)-Y_{i k}(0)\right]\right)+B .
$$

Under Assumptions 1-4 and 6, $\widehat{\beta}_{F E}^{E}$ converges towards the sum of two terms. The first is a weighted average of the LATEs of takers in each waitlist. If those LATEs vary across waitlists, this weighted average is not equal to the LATE of all takers, because it overrepresents waitlists with a ratio of seats to takers closer to $1 / 2{ }^{6}$ The second term, $B$, is a bias term. As explained in Section 2, this bias arises from the endogenous stopping of offers in each waitlist, and from the waitlist fixed effects.

We start by performing comparative statics on $|B|$, assuming that waitlists are homogeneous: there exist real numbers $N_{0}, T_{0}, S_{0}$, and $\Delta_{Y(0)}$ such that for all $k, N_{k}=N_{0}, T_{k}=T_{0}, S_{k}=S_{0}$, and $E\left(\left[\frac{1}{T_{k}} \sum_{i: D_{i k}(1)=1} Y_{i k}(0)-\frac{1}{N_{k}-T_{k}} \sum_{i: D_{i k}(1)=0} Y_{i k}(0)\right]\right)=\Delta_{Y(0)}$. Then,

$$
|B|=\frac{1-t_{0}}{1-s_{0}+N_{0}\left(t_{0}-s_{0}\right)}\left|\Delta_{Y(0)}\right|
$$

where $t_{0}=T_{0} / N_{0}$ and $s_{0}=S_{0} / N_{0}$ respectively denote the proportion of takers and the ratio of seats to applicants in the waitlist. The right hand side of (3) is decreasing in $N_{0},{ }^{7}$ decreasing in $t_{0},{ }^{8}$ increasing in $s_{0},{ }^{9}$ and increasing in $\left|\Delta_{Y(0)}\right|$.

Then, we study how waitlists' heterogeneity affects $|B|$. Let $\left(S_{0}^{a}, S_{0}^{b}\right) \in\left\{2 . . T_{0}-1\right\}^{2}$, let $\left(T_{0}^{a}, T_{0}^{b}\right) \in\left\{3 . . N_{0}\right\}^{2}$, and let $\Delta_{Y(0), k}=E\left[\frac{1}{T_{k}} \sum_{i: D_{i k}(1)=1} Y_{i k}(0)-\frac{1}{N_{k}-T_{k}} \sum_{i: D_{i k}(1)=0} Y_{i k}(0)\right]$. The three following results hold:

1. If $\left(N_{k}, T_{k}, \Delta_{Y(0), k}\right)=\left(N_{0}, T_{0}, \Delta_{Y(0)}\right)$ for all $k,|B|$ is larger if $\alpha \%$ of the waitlists have $S_{0}^{a}$ seats and $(1-\alpha) \%$ have $S_{0}^{b}$ seats than if all of them have $\alpha S_{0}^{a}+(1-\alpha) S_{0}^{b}$ seats.

${ }^{6}$ This can be seen from the fact that $\frac{S_{k}\left(N_{k}-S_{k} \frac{N_{k}+1}{T_{k}+1}\right)}{N_{k}}=T_{k} \frac{S_{k}}{T_{k}}\left(1-\frac{S_{k}}{\left(T_{k}+1\right) N_{k} /\left(N_{k}+1\right)}\right) \approx T_{k} \frac{S_{k}}{T_{k}}\left(1-\frac{S_{k}}{T_{k}}\right)$.

${ }^{7}$ This remains true if the proportion of takers and the ratio of seats to applicants vary across waitlists.

${ }^{8}$ This remains true if the number of applicants and the ratio of seats to applicants vary across waitlists.

${ }^{9}$ This remains true if the number of applicants and the proportion of takers vary across waitlists. 
2. If $\left(N_{k}, S_{k}, \Delta_{Y(0), k}\right)=\left(N_{0}, S_{0}, \Delta_{Y(0)}\right)$ for all $k,|B|$ is larger if $\alpha \%$ of the waitlists have $T_{0}^{a}$ takers and $(1-\alpha) \%$ have $T_{0}^{b}$ takers than if all of them have $\alpha T_{0}^{a}+(1-\alpha) T_{0}^{b}$ takers.

3. If $\left(\frac{T_{k}}{N_{k}}, \frac{S_{k}}{N_{k}}, \Delta_{Y(0), k}\right)=\left(t_{0}, s_{0}, \Delta_{Y(0)}\right)$ for all $k,|B|$ is larger if $\alpha \%$ of the waitlists have $N_{0}^{a}$ applicants and $(1-\alpha) \%$ have $N_{0}^{b}$ applicants than if all have $\alpha N_{0}^{a}+(1-\alpha) N_{0}^{b}$ applicants.

Overall, $|B|$ seems to be higher when waitlists have heterogeneous numbers of applicants, takers, and seats. The impact of waitlists' heterogeneity on $|B|$ can be large. For instance, if $\left(N_{k}, S_{k}, \Delta_{Y(0), k}\right)=\left(40,20, \Delta_{Y(0)}\right),|B|$ is $17.1 \%$ larger if $50 \%$ of waitlists have 25 takers and $50 \%$ have 35 takers than if all have 30 takers.

\subsubsection{Comparison with the Initial Offer estimator}

Let $Z_{i k}^{\prime}=1\left\{R_{i k} \leq S_{k}\right\}$ be an indicator for applicants in the initial round of offers, the so-called initial-offer instrument. Let $S=\sum_{k=1}^{K} S_{k}$. Let $w_{i k}^{I}=Z_{i k}^{\prime} \times \frac{S}{N} \times \frac{N_{k}}{S_{k}}+\left(1-Z_{i k}^{\prime}\right) \times \frac{N-S}{N} \times \frac{N_{k}}{N_{k}-S_{k}}$ be the propensity score weights attached to initial offers. Let $\widehat{\beta}_{P S}^{I}$ be the coefficient of $D_{i k}$ in a 2SLS regression of $Y_{i k}$ on $D_{i k}$, using $Z_{i k}^{\prime}$ as the instrument, and weighted by $w_{i k}^{I}$. We call $\widehat{\beta}_{P S}^{I}$ the IO estimator.

Under Assumptions 1-2 and a technical condition similar to Assumption 4, $\sqrt{K}\left(\widehat{\beta}_{P S}^{I}-\Delta_{K}\right)$ converges towards a normal distribution. Contrary to $Z_{i k}, Z_{i k}^{\prime}$ is only a function of applicants' random numbers and of the number of seats in their waitlist. Thus, it satisfies the random instrument assumption in Imbens \& Angrist (1994). Under Assumption 1, it also satisfies the monotonicity condition therein. Then, one can show that $\widehat{\beta}_{P S}^{I}$ is an asymptotically normal estimator of the LATE of applicants complying with an initial offer. As those are a random subset of the takers, this LATE is equal to $\Delta_{K}$.

However, using $\widehat{\beta}_{P S}^{I}$ instead of $\widehat{\Delta}$ may result in a large loss of precision, as shown in Theorem 3.3 below. For every $k$, let $\mathcal{D}_{k}=\left(D_{1 k}(1), \ldots, D_{N_{k}}(1)\right)$.

Assumption 5 (Assumptions to compare the asymptotic variances of $\widehat{\Delta}$ and $\widehat{\beta}_{P S}^{I}$ )

a) For every $(i, k) \neq\left(i^{\prime}, k^{\prime}\right) \in \mathcal{I}^{2}, \operatorname{cov}\left(Y_{i k}(0), Y_{i^{\prime} k^{\prime}}(0) \mid\left(\mathcal{D}_{k}, \mathcal{R}_{k}\right)_{1 \leq k \leq K}\right)=0$.

b) For every $(i, k) \in \mathcal{I}, V\left(Y_{i k}(0) \mid\left(\mathcal{D}_{k}, \mathcal{R}_{k}\right)_{1 \leq k \leq K}\right)=\sigma_{Y(0)}^{2}$.

c) For every $k \in\{1 . . K\}$, for every $i \in\left\{1 . . N_{k}\right\}, E\left(Y_{i k}(0) \mid\left(\mathcal{D}_{k}, \mathcal{R}_{k}\right)_{1 \leq k \leq K}\right)=\mu_{k}$.

d) For every $(i, k) \in \mathcal{I}, Y_{i k}(1)-Y_{i k}(0)=\tau$.

e) For every $k \in\{1 . . K\}, N_{k}=N_{0}, S_{k}=S_{0}, T_{k}=T_{0}$, for some integers $N_{0}, S_{0}$, and $T_{0}$.

f) $S_{0}\left(N_{0}-S_{0}\right)-N_{0}\left(N_{0}-T_{0}\right)>0$. 
Point a) of Assumption 5 requires that the potential outcomes $Y_{i k}(0)$ be uncorrelated across applicants, point b) requires that they be homoskedastic, and point c) requires that in each waitlist, $E\left(Y_{i k}(0) \mid\left(\mathcal{D}_{k}, \mathcal{R}_{k}\right)_{1 \leq k \leq K}\right)$ be constant. Point d) requires that the treatment effect be constant. Point e) requires that all waitlists have the same number of applicants, takers, and seats. Point $\mathrm{f}$ ) ensures that the denominator of the IO estimator differs from 0 with probability 1. The conditions in Assumption 5 help simplify the formulas of $V(\widehat{\Delta})$ and $V\left(\widehat{\beta}_{P S}^{I}\right)$, thus ensuring that these variances can be compared analytically. Some of these conditions may not be plausible in practice. For instance, point c) fails if the expected value of $Y_{i k}(0)$ differs across takers and non takers. However, in simulations shown in Section 3.2 of the Web appendix, we find that the variance formulas derived in Theorem 3.3 still provide good approximations of $V(\widehat{\Delta})$ and $V\left(\widehat{\beta}_{P S}^{I}\right)$ when points c), d), and f) of Assumption 5 are violated.

\section{Theorem 3.3}

a) If Assumptions 1-4 and a)-e) of Assumption 5 hold,

$$
\limsup _{K \rightarrow+\infty} V\left(\sqrt{K}\left(\widehat{\Delta}-\Delta_{K}\right)\right) \leq \sigma_{Y(0)}^{2} \frac{\frac{1}{S_{0}-1}+\frac{1}{T_{0}-S_{0}}}{\frac{T_{0}}{N_{0}}}
$$

b) If Assumptions 1-5 hold, $\lim _{K \rightarrow+\infty} V\left(\sqrt{K}\left(\widehat{\beta}_{P S}^{I}-\Delta_{K}\right)\right)=\sigma_{Y(0)}^{2} \frac{\frac{1}{S_{0}+\frac{1}{N_{0}-S_{0}}}}{\left(\frac{T_{0}-S_{0}}{N_{0}-S_{0}}\right)^{2}}$.

Point a) of Theorem 3.3 gives an upper bound of the limsup of $V\left(\sqrt{K}\left(\widehat{\Delta}-\Delta_{K}\right)\right)$, ${ }^{10}$ while point b) gives the limit of $V\left(\sqrt{K}\left(\widehat{\beta}_{P S}^{I}-\Delta_{K}\right)\right)$. In order to compare these parameters, note that $0<\frac{T_{0}}{N_{0}} \leq 1-\frac{1}{S_{0}}<1$ is a sufficient condition to have $\frac{\frac{1}{S_{0}-1}+\frac{1}{T_{0}-S_{0}}}{\frac{T_{0}}{N_{0}}} \leq \frac{\frac{1}{S_{0}}+\frac{1}{N_{0}-S_{0}}}{\left(\frac{T_{0}-S_{0}}{N_{0}-S_{0}}\right)^{2}}$. This condition usually holds in practice. For instance, if $\frac{T_{0}}{N_{0}}=0.75$, the condition will hold as soon as there are more than 4 seats per waitlist. Then, the upper bound in point a) is lower than the limit of $V\left(\sqrt{K}\left(\widehat{\beta}_{P S}^{I}-\Delta_{K}\right)\right)$. In practice, using the IO rather than the DREO estimator can lead to large efficiency losses. For instance, if $N_{0}=40, T_{0}=30$, and $S_{0}=20$, the asymptotic variance of the IO estimator is 1.97 times larger than that of the DREO one.

\section{Application to Blattman \& Annan (2016)}

After the second Liberian civil war, some ex-fighters started engaging in illegal activities, and working abroad as mercenaries. Blattman \& Annan (2016) ${ }^{11}$ study the effect of an agricultural training on their employment and on their social networks. By improving their labor market

\footnotetext{
${ }^{10}$ Under technical conditions, for instance if one assumes that the potential outcomes have a bounded support, it follows from Theorem 2.20 in Van der Vaart (2000) and Theorem 3.1 that $\sqrt{K}\left(\widehat{\Delta}-\Delta_{K}\right)$ converges in $L^{2}$, so $\limsup _{K \rightarrow+\infty} V\left(\sqrt{K}\left(\widehat{\Delta}-\Delta_{K}\right)\right)$ is actually a simple limit, and it is equal to $\sigma^{2}$, the asymptotic variance of $\widehat{\Delta}$.

${ }^{11}$ Blattman \& Annan (2016) is one of the few articles in our survey in Section 1 of the Web appendix whose data is not proprietary.
} 
opportunities, the program hoped to reduce their interest in illegal and mercenary activities, and to sever their relationships with other ex-combatants. To allocate the treatment, the authors divided applicants into 70 waitlists, according to the training site they applied for, their former military rank, and their community. In each waitlist, they randomly ranked applicants, and offers were made following that ranking until the seats available were filled.

Blattman \& Annan (2016) estimate the training's effect on 62 outcomes, that are either applicants' answers to survey questions, or indexes averaging their answers to several related questions. To preserve space, we only consider the main outcomes. Here are the rules we used to make our selection: we chose indexes rather than questions averaged into an index; among questions not averaged into an index, we discarded those asking applicants to give a subjective opinion; finally, we discarded a few measures the authors did not comment on in the paper. We end up with four measures of employment, one measure of applicants' interest in working as mercenaries, and five measures of their social network.

For each outcome, Table 3 below shows the EO estimator computed by the authors, and the DREO estimator computed with the same controls as those used by the authors. ${ }^{12}$ An estimate of $\widehat{\sigma}_{+} / \sqrt{K-1}$ is shown next to each DREO estimator. ${ }^{13}$ In Theorem 2.1 in the Web appendix, we derive the asymptotic distribution of the EO estimator. Accordingly, an estimate of $\widehat{\sigma}_{E,+}^{2} / \sqrt{K-1}$, defined in Section 2.1 of the Web appendix, is shown next to each EO estimator. The table then shows the p-value of a t-test that the EO and DREO estimators are equal, also computed following Theorem 2.1 in the Web appendix. Finally, the table shows the estimated difference between the mean of $Y_{i k}(0)$ among non-takers and takers, denoted $\Delta_{Y(0)}$. The EO and DREO estimators are close for all employment outcomes, but they significantly differ for three of the other outcomes. For applicants' interest in mercenary work, the DREO estimator is $51.0 \%$ larger in absolute value than the EO one, and it is statistically significant while the EO estimator is only marginally significant; for applicants' relations with their ex-commanders, the DREO estimator is $47.4 \%$ larger, and it is statistically significant while the EO estimator is not; for applicants' social network quality, the DREO estimator is three times larger, but none of the two estimators is significant. For the first two outcomes, the estimated difference between the mean of $Y_{i k}(0)$ of takers and non-takers is large $(30.7 \%$ and $25.1 \%$ of the standard deviation of these variables, respectively), which may explain why the EO and DREO estimators differ.

\footnotetext{
${ }^{12}$ The DREO estimator with controls is defined in Section 2.4 of the Web Appendix.

${ }^{13}$ To account for the controls included in the estimation, $Y_{i k}$ and $D_{i k}$ are regressed on the controls, and then the residuals from those two regressions are used instead of $Y_{i k}$ and $D_{i k}$ in the computation of $\widehat{\sigma}_{+}$.
} 
Table 3: Estimators of the LATE in Blattman \& Annan (2016)

\begin{tabular}{lcccc}
\hline \hline & EO (s.e.) & DREO (s.e.) & EO=DREO & $\Delta_{Y(0)}$ \\
\hline Works in agriculture & $0.155(0.037)$ & $0.167(0.037)$ & 0.214 & 0.020 \\
Hours illegal work & $-3.697(1.822)$ & $-3.188(1.614)$ & 0.183 & -2.807 \\
Hours farming work & $4.090(1.511)$ & $4.319(1.472)$ & 0.468 & 3.070 \\
Income index & $0.157(0.075)$ & $0.169(0.069)$ & 0.400 & -0.087 \\
Interest mercenary work & $-0.239(0.140)$ & $-0.361(0.155)$ & 0.010 & 0.307 \\
Relations ex-combatants & $0.073(0.091)$ & $0.050(0.097)$ & 0.388 & -0.079 \\
Relations ex-commanders & $-0.154(0.113)$ & $-0.227(0.109)$ & 0.011 & 0.251 \\
Social network quality & $0.027(0.068)$ & $0.082(0.068)$ & 0.013 & -0.041 \\
Social support & $0.188(0.091)$ & $0.161(0.089)$ & 0.166 & -0.165 \\
Relationships families & $0.133(0.075)$ & $0.161(0.079)$ & 0.205 & -0.059 \\
N & 1,025 & 1,016 & & \\
\hline \hline
\end{tabular}

Notes. Columns 2 and 3 show the EO and DREO estimators in Blattman \& Annan (2016), for the outcome variables in Column 1, and with the same controls as in Blattman \& Annan (2016). The EO estimators are computed using all the waitlists, while the DREO estimators are computed excluding two waitlists that had less than two seats. An estimate of $\widehat{\sigma}_{+} / \sqrt{K-1}$ is shown next to each DREO estimator, between parentheses. An estimate of $\widehat{\sigma}_{E,+}^{2} / \sqrt{K-1}$ (see Section 2.1 of the Web appendix) is shown next to each EO estimator, between parentheses. Column 4 shows the p-value of a t-test that the EO and DREO estimators are equal, where we follow Theorem 2.1 in the Web appendix to compute the standard error of the difference between the two estimators. Column 5 shows the estimated difference between the mean of $Y_{i k}(0)$ among takers and non-takers.

\section{Conclusion}

When the seats available for a treatment are allocated using randomized waitlists, we show that applicants getting and not getting an offer are not statistically comparable. Accordingly, a commonly used estimator of the treatment effect, the ever-offer estimator, is inconsistent when the number of waitlists goes to infinity. We propose a new estimator, the doublyreweighted ever-offer (DREO) estimator, and we show that it is consistent and asymptotically normal. Finally, we show that the DREO estimator is often more efficient than another consistent estimator, the initial-offer estimator. Overall, we recommend that practitioners use the DREO estimator when they analyze randomized waitlists. 


\section{References}

Abadie, A., Angrist, J. \& Imbens, G. (2002), 'Instrumental variables estimates of the effect of subsidized training on the quantiles of trainee earnings', Econometrica 70(1), 91-117.

Angrist, J. D., Imbens, G. W. \& Rubin, D. B. (1996), 'Identification of causal effects using instrumental variables', Journal of the American Statistical Association 91(434), pp. 444455.

Angrist, J. D. \& Pischke, J.-S. (2008), Mostly harmless econometrics: An empiricist's companion, Princeton university press.

Behaghel, L., de Chaisemartin, C. \& Gurgand, M. (2017), 'Ready for boarding? the effects of a boarding school for disadvantaged students', American Economic Journal: Applied Economics 9(1), 140-164.

Billingsley, P. (1986), Probability and measure, 2nd edn, John Wiley \& Sons.

Blattman, C. \& Annan, J. (2016), 'Can employment reduce lawlessness and rebellion? a field experiment with high-risk men in a fragile state', American Political Science Review $\mathbf{1 1 0}(1), 1-17$.

Crépon, B., Devoto, F., Duflo, E. \& Parienté, W. (2015), 'Estimating the impact of microcredit on those who take it up: Evidence from a randomized experiment in morocco', American Economic Journal: Applied Economics 7(1), 123-150.

de Chaisemartin, C. \& D'Haultfouille, X. (2018), 'Fuzzy differences-in-differences', The Review of Economic Studies 85(2), 999-1028.

Gut, A. (1992), 'The weak law of large numbers for arrays', Statistics 83 probability letters $\mathbf{1 4}(1), 49-52$.

Imbens, G. W. \& Angrist, J. D. (1994), 'Identification and estimation of local average treatment effects', Econometrica 62(2), pp. 467-475.

Imbens, G. W. \& Rosenbaum, P. R. (2005), 'Robust, accurate confidence intervals with a weak instrument: quarter of birth and education', Journal of the Royal Statistical Society: Series A (Statistics in Society) 168(1), 109-126.

Liu, R. Y. \& Singh, K. (1995), 'Using iid bootstrap inference for general non-iid models', Journal of statistical planning and inference 43(1-2), 67-75.

Neyman, J. (1923), 'On the application of probability theory to agricultural experiments. essay on principles. section 9'. translated in Statistical Science 5(4), 465-472, 1990.

Van der Vaart, A. W. (2000), Asymptotic statistics, Vol. 3, Cambridge university press. 


\section{A Proofs}

The next lemma shows that the expectation of the average of any function of potential treatments and outcomes is the same among $w_{i k}$-reweighted applicants getting an offer and those not getting an offer. $\forall(i, k) \in \mathcal{I}$, let $P_{i k}=\left(D_{i k}(1), Y_{i k}(0), Y_{i k}(1)\right)$.

Lemma A.1 If Assumptions 1-3 hold, then $\forall k \in\{1 . . K\}$ and for any function $\phi: \mathbb{R}^{3} \mapsto \mathbb{R}$,

$E\left(\frac{1}{L_{k}-1} \sum_{i: Z_{i k}=1} w_{i k} \phi\left(P_{i k}\right) \mid \mathcal{P}_{k}\right)=E\left(\frac{1}{N_{k}-L_{k}} \sum_{i: Z_{i k}=0} \phi\left(P_{i k}\right) \mid \mathcal{P}_{k}\right)=\frac{1}{N_{k}} \sum_{i=1}^{N_{k}} \phi\left(P_{i k}\right)$.

\section{Proof of Lemma A.1}

We start by showing that

$$
E\left(\frac{1}{L_{k}-1} \sum_{i: Z_{i k}=1} w_{i k} \phi\left(P_{i k}\right) \mid \mathcal{P}_{k}\right)=\frac{1}{N_{k}} \sum_{i=1}^{N_{k}} \phi\left(P_{i k}\right)
$$

First, we show that (4) holds when $\mathcal{P}_{k}$ is such that $T_{k}<N_{k}$. Then, we have

$$
\begin{aligned}
& E\left(\frac{1}{L_{k}-1} \sum_{i: Z_{i k}=1} w_{i k} \phi\left(P_{i k}\right) \mid \mathcal{P}_{k}\right) \\
& =E\left(\sum_{i=1}^{N_{k}} \frac{1}{L_{k}-1}\left(1-\frac{D_{i k}(1)}{S_{k}}\right) \phi\left(P_{i k}\right) 1\left\{R_{i k} \leq L_{k}\right\} \mid \mathcal{P}_{k}\right) \\
& =\sum_{i=1}^{N_{k}}\left(1-\frac{D_{i k}(1)}{S_{k}}\right) \phi\left(P_{i k}\right) E\left(\frac{1}{L_{k}-1} 1\left\{R_{i k} \leq L_{k}\right\} \mid \mathcal{P}_{k}\right) \\
& =\sum_{i=1}^{N_{k}}\left(1-\frac{D_{i k}(1)}{S_{k}}\right) \phi\left(P_{i k}\right) \sum_{l=S_{k}}^{N_{k}-T_{k}+S_{k}} P\left(L_{k}=l \mid \mathcal{P}_{k}\right) \frac{1}{l-1} E\left(1\left\{R_{i k} \leq l\right\} \mid L_{k}=l, \mathcal{P}_{k}\right) \\
& =\sum_{i=1}^{N_{k}}\left(1-\frac{D_{i k}(1)}{S_{k}}\right) \phi\left(P_{i k}\right) \sum_{l=S_{k}}^{N_{k}-T_{k}+S_{k}} \frac{\left(\begin{array}{c}
l-1 \\
S_{k}-1
\end{array}\right)\left(\begin{array}{c}
N_{k}-l \\
T_{k}-S_{k}
\end{array}\right)}{\left(\begin{array}{c}
N_{k} \\
T_{k}
\end{array}\right)} \frac{1}{l-1} E\left(1\left\{R_{i k} \leq l\right\} \mid L_{k}=l, \mathcal{P}_{k}\right) \\
& =\sum_{i=1}^{N_{k}}\left(1-\frac{D_{i k}(1)}{S_{k}}\right) \phi\left(P_{i k}\right) \sum_{l=S_{k}}^{N_{k}-T_{k}+S_{k}} \frac{\left(\begin{array}{c}
l-1 \\
S_{k}-1
\end{array}\right)\left(\begin{array}{c}
N_{k}-l \\
T_{k}-S_{k}
\end{array}\right)}{\left(\begin{array}{l}
N_{k} \\
T_{k}
\end{array}\right)} \frac{1}{l-1}\left(D_{i k}(1) \frac{S_{k}}{T_{k}}+\left(1-D_{i k}(1)\right) \frac{l-S_{k}}{N_{k}-T_{k}}\right) \\
& =\frac{1}{N_{k}} \sum_{i=1}^{N_{k}} \phi\left(P_{i k}\right)\left(D_{i k}(1) \sum_{l=S_{k}}^{N_{k}-T_{k}+S_{k}} \frac{\left(\begin{array}{c}
l-1 \\
S_{k}-1
\end{array}\right)\left(\begin{array}{c}
N_{k}-l \\
T_{k}-S_{k}
\end{array}\right) \frac{S_{k}-1}{l-1}}{\left(\begin{array}{c}
N_{k} \\
T_{k}
\end{array}\right) \frac{T_{k}}{N_{k}}}+\left(1-D_{i k}(1)\right) \sum_{l=S_{k}+1}^{N_{k}-T_{k}+S_{k}} \frac{\left(\begin{array}{c}
l-1 \\
S_{k}-1
\end{array}\right)\left(\begin{array}{c}
N_{k}-l \\
T_{k}-S_{k}
\end{array}\right) \frac{l-S_{k}}{l-1}}{\left(\begin{array}{c}
N_{k} \\
T_{k}
\end{array}\right) \frac{N_{k}-T_{k}}{N_{k}}}\right) \\
& =\frac{1}{N_{k}} \sum_{i=1}^{N_{k}} \phi\left(P_{i k}\right)\left(D_{i k}(1) \sum_{l=S_{k}-1}^{N_{k}-T_{k}+S_{k}-1} \frac{\left(\begin{array}{c}
l-1 \\
S_{k}-2
\end{array}\right)\left(\begin{array}{c}
N_{k}-1-l \\
T_{k}-S_{k}
\end{array}\right)}{\left(\begin{array}{c}
N_{k}-1 \\
T_{k}-1
\end{array}\right)}+\left(1-D_{i k}(1)\right) \sum_{l=S_{k}}^{N_{k}-1-T_{k}+S_{k}} \frac{\left(\begin{array}{c}
l-1 \\
S_{k}-1
\end{array}\right)\left(\begin{array}{c}
N_{k}-1-l \\
T_{k}-S_{k}
\end{array}\right)}{\left(\begin{array}{c}
N_{k}-1 \\
T_{k}
\end{array}\right)}\right) \\
& =\frac{1}{N_{k}} \sum_{i=1}^{N_{k}} \phi\left(P_{i k}\right) \text {. }
\end{aligned}
$$


The first equality follows from the definitions of $w_{i k}, Z_{i k}$, and $D_{i k}$. The second equality holds because $D_{i k}(1)$ and $\phi\left(P_{i k}\right)$ are functions of $\mathcal{P}_{k}, N_{k}$ and $S_{k}$ are non stochastic, and the conditional expectation is linear. The third follows from the law of iterated expectations, and the fact that $L_{k}$ is included between $S_{k}$ and $N_{k}-T_{k}+S_{k}$ under Assumptions 1 and 3 .

Then, under Assumption 1, having $L_{k}=l$ is equivalent to having $S_{k}-1$ takers with $R_{i k} \leq l-1$, one with $R_{i k}=l$, and $T_{k}-S_{k}$ with $R_{i k} \geq l+1$. $\left(\begin{array}{c}l-1 \\ S_{k}-1\end{array}\right)\left(\begin{array}{c}N_{k}-l \\ T_{k}-S_{k}\end{array}\right) T_{k} !\left(N_{k}-T_{k}\right)$ ! possible values of $\mathcal{R}_{k}$ satisfy these constraints. Under Assumption 2, conditional on $\mathcal{P}_{k}$ each of those values has a probability $\frac{1}{N_{k} \text { ! }}$ of being realized. Hence the fourth equality.

Then,

$$
\begin{aligned}
E\left(1\left\{R_{i k} \leq l\right\} \mid L_{k}=l, \mathcal{P}_{k}\right) & =D_{i k}(1) E\left(1\left\{R_{i k} \leq l\right\} \mid L_{k}=l, D_{i k}(1)=1, \mathcal{P}_{k} \backslash D_{i k}(1)\right) \\
& +\left(1-D_{i k}(1)\right) E\left(1\left\{R_{i k} \leq l\right\} \mid L_{k}=l, D_{i k}(1)=0, \mathcal{P}_{k} \backslash D_{i k}(1)\right)
\end{aligned}
$$

Conditional on $L_{k}=l, S_{k}$ takers out of $T_{k}$ satisfy $R_{i k} \leq l$, and Assumption 2 ensures that each taker has the same probability of satisfying this condition, so

$$
E\left(1\left\{R_{i k} \leq l\right\} \mid L_{k}=l, D_{i k}(1)=1, \mathcal{P}_{k} \backslash D_{i k}(1)\right)=\frac{S_{k}}{T_{k}} .
$$

Similarly, conditional on $L_{k}=l$ and $T_{k}<N_{k}, l-S_{k}$ non-takers out of $N_{k}-T_{k}$ satisfy $R_{i k} \leq l$, and Assumption 2 ensures that each has the same probability of satisfying this condition, so

$$
E\left(1\left\{R_{i k} \leq l\right\} \mid L_{k}=l, D_{i k}(1)=0, \mathcal{P}_{k} \backslash D_{i k}(1)\right)=\frac{l-S_{k}}{N_{k}-T_{k}} .
$$

Plugging (7) and (8) into (6) yields the fifth equality. The sixth and seventh equalities follow after some algebra.

Then, we prove the eighth equality. Before that, note that $T_{k}<N_{k}$ and Assumption 3 ensure that $1 \leq S_{k}-1 \leq T_{k}-1 \leq N_{k}-1$ and $1 \leq S_{k} \leq T_{k} \leq N_{k}-1$, thus ensuring that all the quantities that follow are well-defined. There are $\left(\begin{array}{l}N_{k}-1 \\ T_{k}-1\end{array}\right)$ ways of distributing $T_{k}-1$ units over $N_{k}-1$ ranks. The rank of the $S_{k}-1$ th unit must be included between $S_{k}-1$ and $N_{k}-T_{k}+S_{k}-1$, and for every $l \in\left\{S_{k}-1 . . N_{k}-T_{k}+S_{k}-1\right\}$, there are $\left(\begin{array}{c}l-1 \\ S_{k}-2\end{array}\right)\left(\begin{array}{c}N_{k}-1-l \\ T_{k}-S_{k}\end{array}\right)$ ways of distributing those $T_{k}-1$ units while having that the $S_{k}-1$ th unit is at the $l$ th rank. Therefore,

$$
\sum_{l=S_{k}-1}^{N_{k}-T_{k}+S_{k}-1}\left(\begin{array}{c}
l-1 \\
S_{k}-2
\end{array}\right)\left(\begin{array}{c}
N_{k}-1-l \\
T_{k}-S_{k}
\end{array}\right)=\left(\begin{array}{c}
N_{k}-1 \\
T_{k}-1
\end{array}\right) .
$$

Similarly, when distributing $T_{k}$ units over $N_{k}-1$ ranks, the rank of the $S_{k}$ th unit must lie between $S_{k}$ and $N_{k}-1-T_{k}+S_{k}$. For every $l \in\left\{S_{k} . . N_{k}-1-T_{k}+S_{k}\right\}$, there are $\left(\begin{array}{c}l-1 \\ S_{k}-1\end{array}\right)\left(\begin{array}{c}N_{k}-1-l \\ T_{k}-S_{k}\end{array}\right)$ ways of distributing those $T_{k}$ units while having the $S_{k}$ th unit at the $l$ th rank. Thus,

$$
\sum_{l=S_{k}}^{N_{k}-1-T_{k}+S_{k}}\left(\begin{array}{c}
l-1 \\
S_{k}-1
\end{array}\right)\left(\begin{array}{c}
N_{k}-1-l \\
T_{k}-S_{k}
\end{array}\right)=\left(\begin{array}{c}
N_{k}-1 \\
T_{k}
\end{array}\right) .
$$


The eighth equality follows from (9) and (10). This concludes the proof of (5).

Second, we show that (4) holds when $\mathcal{P}_{k}$ is such that $T_{k}=N_{k}$. Then, we have

$$
\begin{aligned}
E\left(\frac{1}{L_{k}-1} \sum_{i: Z_{i k}=1} w_{i k} \phi\left(P_{i k}\right) \mid \mathcal{P}_{k}\right) & =E\left(\sum_{i=1}^{N_{k}} \phi\left(P_{i k}\right) \frac{1}{S_{k}} 1\left\{R_{i k} \leq S_{k}\right\} \mid \mathcal{P}_{k}\right) \\
& =\sum_{i=1}^{N_{k}} \phi\left(P_{i k}\right) \frac{1}{S_{k}} E\left(1\left\{R_{i k} \leq S_{k}\right\} \mid \mathcal{P}_{k}\right) \\
& =\frac{1}{N_{k}} \sum_{i=1}^{N_{k}} \phi\left(P_{i k}\right) .
\end{aligned}
$$

The first equality follows from the definition of $w_{i k}$ and from the fact that if $T_{k}=N_{k}, L_{k}=S_{k}$. The second equality holds because $\phi\left(P_{i k}\right)$ is a function of $\mathcal{P}_{k}, N_{k}$ and $S_{k}$ are non stochastic, and the conditional expectation is linear. The third equality follows from the fact that under Assumption 2, if $T_{k}=N_{k}$ then conditional on $\mathcal{P}_{k}$ each applicant has a probability $\frac{S_{k}}{N_{k}}$ of having $R_{i k} \leq S_{k}$. This proves (11). (5) and (11) prove (4).

We then show that

$$
E\left(\frac{1}{N_{k}-L_{k}} \sum_{i: Z_{i k}=0} \phi\left(P_{i k}\right) \mid \mathcal{P}_{k}\right)=\frac{1}{N_{k}} \sum_{i=1}^{N_{k}} \phi\left(P_{i k}\right) .
$$

First, we show that (12) holds when $\mathcal{P}_{k}$ is such that $T_{k}<N_{k}$. Then, we have

$$
\begin{aligned}
& E\left(\frac{1}{N_{k}-L_{k}} \sum_{i: Z_{i k}=0} \phi\left(P_{i k}\right) \mid \mathcal{P}_{k}\right) \\
& =\sum_{i=1}^{N_{k}} \phi\left(P_{i k}\right) E\left(\frac{1}{N_{k}-L_{k}} 1\left\{R_{i k}>L_{k}\right\} \mid \mathcal{P}_{k}\right) \\
& =\sum_{i=1}^{N_{k}} \phi\left(P_{i k}\right) \sum_{l=S_{k}}^{N_{k}-T_{k}+S_{k}} \frac{\left(\begin{array}{c}
l-1 \\
S_{k}-1
\end{array}\right)\left(\begin{array}{c}
N_{k}-l \\
T_{k}-S_{k}
\end{array}\right)}{\left(\begin{array}{c}
N_{k} \\
T_{k}
\end{array}\right)} \frac{1}{N_{k}-l} E\left(1\left\{R_{i k}>l\right\} \mid L_{k}=l, \mathcal{P}_{k}\right) \\
& =\sum_{i=1}^{N_{k}} \phi\left(P_{i k}\right) \sum_{l=S_{k}}^{N_{k}-T_{k}+S_{k}} \frac{\left(\begin{array}{c}
l-1 \\
S_{k}-1
\end{array}\right)\left(\begin{array}{c}
N_{k}-l \\
T_{k}-S_{k}
\end{array}\right)}{\left(\begin{array}{c}
N_{k} \\
T_{k}
\end{array}\right)} \frac{1}{N_{k}-l}\left(D_{i k}(1) \frac{T_{k}-S_{k}}{T_{k}}+\left(1-D_{i k}(1)\right) \frac{N_{k}-T_{k}-l+S_{k}}{N_{k}-T_{k}}\right) \\
& =\frac{1}{N_{k}} \sum_{i=1}^{N_{k}} \phi\left(P_{i k}\right)\left(D_{i k}(1) \sum_{l=S_{k}}^{N_{k}-T_{k}+S_{k}} \frac{\left(\begin{array}{c}
l-1 \\
S_{k}-1
\end{array}\right)\left(\begin{array}{c}
N_{k}-l \\
T_{k}-S_{k}
\end{array}\right) \frac{T_{k}-S_{k}}{N_{k}-l}}{\left(\begin{array}{c}
N_{k} \\
T_{k}
\end{array}\right) \frac{T_{k}}{N_{k}}}+\left(1-D_{i k}(1)\right) \sum_{l=S_{k}}^{N_{k}-1-T_{k}+S_{k}} \frac{\left(\begin{array}{c}
l-1 \\
S_{k}-1
\end{array}\right)\left(\begin{array}{c}
N_{k}-l \\
T_{k}-S_{k}
\end{array}\right) \frac{N_{k}-T_{k}-l+S_{k}}{N_{k}-l}}{\left(\begin{array}{c}
N_{k} \\
T_{k}
\end{array}\right) \frac{N_{k}-T_{k}}{N_{k}}}\right) \\
& =\frac{1}{N_{k}} \sum_{i=1}^{N_{k}} \phi\left(P_{i k}\right)\left(D_{i k}(1) \sum_{l=S_{k}}^{N_{k}-T_{k}+S_{k}} \frac{\left(\begin{array}{c}
l-1 \\
S_{k}-1
\end{array}\right)\left(\begin{array}{c}
N_{k}-1-l \\
T_{k}-1-S_{k}
\end{array}\right)}{\left(\begin{array}{c}
N_{k}-1 \\
T_{k}-1
\end{array}\right)}+\left(1-D_{i k}(1)\right) \sum_{l=S_{k}}^{N_{k}-1-T_{k}+S_{k}} \frac{\left(\begin{array}{c}
l-1 \\
S_{k}-1
\end{array}\right)\left(\begin{array}{c}
N_{k}-1-l \\
T_{k}-S_{k}
\end{array}\right)}{\left(\begin{array}{c}
N_{k}-1 \\
T_{k}
\end{array}\right)}\right) \\
& =\frac{1}{N_{k}} \sum_{i=1}^{N_{k}} \phi\left(P_{i k}\right) \text {. }
\end{aligned}
$$

This derivation follows from arguments similar to those used when deriving (5). We only prove the last equality. Note that Assumption 3 ensures that $1 \leq S_{k} \leq T_{k}-1 \leq N_{k}-1$, 
thus ensuring that all the quantities that follow are well-defined. There are $\left(\begin{array}{l}N_{k}-1 \\ T_{k}-1\end{array}\right)$ ways of distributing $T_{k}-1$ units over $N_{k}-1$ ranks. The rank of the $S_{k}$ th unit must be included between $S_{k}$ and $N_{k}-T_{k}+S_{k}$, and for every $l \in\left\{S_{k} . . N_{k}-T_{k}+S_{k}\right\}$, there are $\left(\begin{array}{c}l-1 \\ S_{k}-1\end{array}\right)\left(\begin{array}{c}N_{k}-1-l \\ T_{k}-1-S_{k}\end{array}\right)$ ways of distributing those $T_{k}-1$ units while having that the $S_{k}$ th unit is at the $l$ th rank. Therefore,

$$
\sum_{l=S_{k}}^{N_{k}-T_{k}+S_{k}}\left(\begin{array}{c}
l-1 \\
S_{k}-1
\end{array}\right)\left(\begin{array}{c}
N_{k}-1-l \\
T_{k}-1-S_{k}
\end{array}\right)=\left(\begin{array}{c}
N_{k}-1 \\
T_{k}-1
\end{array}\right)
$$

The last equality in the derivation of (13) follows from (10) and (14).

Second, we show that (12) holds when $\mathcal{P}_{k}$ is such that $T_{k}=N_{k}$. Then, we have

$$
E\left(\frac{1}{N_{k}-L_{k}} \sum_{i: Z_{i k}=0} \phi\left(P_{i k}\right) \mid \mathcal{P}_{k}\right)=\sum_{i=1}^{N_{k}} \phi\left(P_{i k}\right) \frac{1}{N_{k}-S_{k}} E\left(1\left\{R_{i k}>S_{k}\right\} \mid \mathcal{P}_{k}\right)=\frac{1}{N_{k}} \sum_{i=1}^{N_{k}} \phi\left(P_{i k}\right) .
$$

This derivation follows from arguments similar to those used when deriving (11). (13) and (15) prove (12). QED.

\section{Proof of Lemma 3.1}

We only prove point a), point b) follows from a similar argument.

$$
\begin{aligned}
& E\left(\frac{1}{K} \sum_{k=1}^{K} \frac{N_{k}}{\bar{N}}\left(\frac{1}{L_{k}-1} \sum_{i: Z_{i k}=1} w_{i k} Y_{i k}-\frac{1}{N_{k}-L_{k}} \sum_{i: Z_{i k}=0} Y_{i k}\right)\right) \\
= & \frac{1}{K} \sum_{k=1}^{K} \frac{N_{k}}{\bar{N}} E\left(E\left(\frac{1}{L_{k}-1} \sum_{i: Z_{i k}=1} w_{i k} Y_{i k}\left(D_{i k}(1)\right) \mid \mathcal{P}_{k}\right)-E\left(\frac{1}{N_{k}-L_{k}} \sum_{i: Z_{i k}=0} Y_{i k}(0) \mid \mathcal{P}_{k}\right)\right) \\
= & \frac{1}{K} \sum_{k=1}^{K} \frac{N_{k}}{\bar{N}} E\left(\frac{1}{N_{k}} \sum_{i=1}^{N_{k}}\left[Y_{i k}\left(D_{i k}(1)\right)-Y_{i k}(0)\right]\right) \\
= & E\left(\frac{1}{N} \sum_{(i, k) \in \mathcal{I}}\left[Y_{i k}\left(D_{i k}(1)\right)-Y_{i k}(0)\right]\right) .
\end{aligned}
$$

The first equality follows from the linearity of the expectation, from the fact $N_{k}$ and $\bar{N}$ are not stochastic, from point c) of Assumption 1 and the definitions of $Y_{i k}$ and $D_{i k}$, from the law of iterated expectations, and from the linearity of the conditional expectation. The second equality follows from Lemma A.1, with $\phi\left(P_{i k}\right)=Y_{i k}\left(D_{i k}(1)\right)$ for the first conditional expectation, and $\phi\left(P_{i k}\right)=Y_{i k}(0)$ for the second one. The third equality follows after some algebra. QED.

The proof of Theorem 3.1 below makes use of the following lemma, where $O_{p}(1)$ (resp. $o_{p}(1)$ ) stands for a sequence of random variables bounded in probability (resp. converging towards 0 in probability), see, e.g., Van der Vaart (2000). 
Lemma A.2 Let $\left(A_{K}\right)_{K \in \mathbb{N}}$ and $\left(B_{K}\right)_{K \in \mathbb{N}}$ be two sequences of real numbers such that for every $K, B_{K} \geq C$ for some real number $C>0$, and $\frac{A_{K}}{B_{K}}$ converges towards a finite limit. Let $\left(\widehat{A}_{K}\right)_{K \in \mathbb{N}}$ and $\left(\widehat{B}_{K}\right)_{K \in \mathbb{N}}$ be two sequences of random variables such that $\sqrt{K}\left(\widehat{A}_{K}-A_{K}\right)=$ $O_{p}(1)$ and $\sqrt{K}\left(\widehat{B}_{K}-B_{K}\right)=O_{p}(1)$. Then,

$$
\sqrt{K}\left(\frac{\widehat{A}_{K}}{\widehat{B}_{K}}-\frac{A_{K}}{B_{K}}\right)=\sqrt{K} \frac{1}{B_{K}}\left(\left(\widehat{A}_{K}-A_{K}\right)-\frac{A_{K}}{B_{K}}\left(\widehat{B}_{K}-B_{K}\right)\right)+o_{P}(1) .
$$

\section{Proof of Lemma A.2}

$\sqrt{K}\left(\widehat{A}_{K}-A_{K}\right)=O_{p}(1)$ and $\sqrt{K}\left(\widehat{B}_{K}-B_{K}\right)=O_{p}(1)$ imply that $\widehat{A}_{K}-A_{K}=o_{p}(1)$ and $\widehat{B}_{K}-B_{K}=o_{p}(1)$. Therefore, with probability approaching one, $\max \left(\widehat{A}_{K}-A_{K}, \widehat{B}_{K}-B_{K}\right) \leq$ $\frac{C}{2}$. Then, Lemma S3 in de Chaisemartin \& D'Haultfouille (2018) implies that with probability approaching one,

$$
\begin{aligned}
& \left|\sqrt{K}\left(\frac{\widehat{A}_{K}}{\widehat{B}_{K}}-\frac{A_{K}}{B_{K}}\right)-\sqrt{K} \frac{1}{B_{K}}\left(\left(\widehat{A}_{K}-A_{K}\right)-\frac{A_{K}}{B_{K}}\left(\widehat{B}_{K}-B_{K}\right)\right)\right| \\
\leq & \frac{2\left(1+\frac{A_{K}}{B_{K}}\right)}{C^{2}} \max \left(\sqrt{K}\left(\widehat{A}_{K}-A_{K}\right), \sqrt{K}\left(\widehat{B}_{K}-B_{K}\right)\right) \max \left(\widehat{A}_{K}-A_{K}, \widehat{B}_{K}-B_{K}\right) .
\end{aligned}
$$

The right hand side of the inequality in the previous display is an $o_{p}(1)$. With probability approaching one, the left hand side is bounded by an $o_{p}(1)$, so it is itself an $o_{p}(1)$. QED.

\section{Proof of Theorem 3.1}

Proof that $\sqrt{K}\left(\widehat{\Delta}-\Delta_{K}\right) \stackrel{d}{\longrightarrow} \mathcal{N}\left(0, \sigma^{2}\right)$

First, notice that

$$
\begin{aligned}
\Delta_{K} & =\frac{1}{T} E\left(\sum_{(i, k): D_{i k}(1)=1}\left[Y_{i k}(1)-Y_{i k}(0)\right]\right) \\
& =\frac{E\left(\frac{1}{N} \sum_{(i, k) \in \mathcal{I}}\left[Y_{i k}\left(D_{i k}(1)\right)-Y_{i k}(0)\right]\right)}{\frac{1}{N} \sum_{(i, k) \in \mathcal{I}} D_{i k}(1)} \\
& =\frac{E\left(\frac{1}{K} \sum_{k=1}^{K} R F_{k}\right)}{E\left(\frac{1}{K} \sum_{k=1}^{K} F S_{k}\right)} .
\end{aligned}
$$

The first equality follows from point b) of Assumption 4. The second equality follows from some algebra, and from point a) of Assumption 1. The last equality follows from points a) and b) of Lemma 3.1, point b) of Assumption 4, and the definitions of $R F_{k}$ and $F S_{k}$. 
Then,

$$
\begin{aligned}
& \sqrt{K}\left(\frac{1}{K} \sum_{k=1}^{K} R F_{k}-E\left(\frac{1}{K} \sum_{k=1}^{K} R F_{k}\right)\right) \\
= & \frac{\sum_{k=1}^{K}\left(R F_{k}-E\left(R F_{k}\right)\right)}{\sqrt{\sum_{k=1}^{K} V\left(R F_{k}\right)}} \sqrt{\frac{1}{K} \sum_{k=1}^{K} V\left(R F_{k}\right) .}
\end{aligned}
$$

Point a) of Assumption 1 and point a) of Assumption 4 ensures that the $R F_{k}$ s are independent. Point d) of Assumption 4 ensures that for every $k$, the expectation and variance of $R F_{k}$ exist, and points e.iii) and e.vi) ensures that $\left(R F_{k}\right)_{k \in \mathbb{N}^{*}}$ satisfies the Liapunov condition. Then, the Liapunov central limit theorem (see, e.g., Billingsley, 1986, Theorem 27.3) implies that

$$
\frac{\sum_{k=1}^{K}\left(R F_{k}-E\left(R F_{k}\right)\right)}{\sqrt{\sum_{k=1}^{K} V\left(R F_{k}\right)}} \stackrel{d}{\longrightarrow} \mathcal{N}(0,1) .
$$

Point e.iii) of Assumption 4 ensures that $\sqrt{\frac{1}{K} \sum_{k=1}^{K} V\left(R F_{k}\right)}$ has a finite limit, denoted $\sigma_{R F}$. Therefore, combining (17), (18), and the Slutsky lemma,

$$
\sqrt{K}\left(\frac{1}{K} \sum_{k=1}^{K}\left(R F_{k}-E\left(\frac{1}{K} \sum_{k=1}^{K} R F_{k}\right)\right)\right) \stackrel{d}{\longrightarrow} \mathcal{N}\left(0, \sigma_{R F}^{2}\right) .
$$

Similarly, let $\sigma_{F S}$ be the limit of $\sqrt{\frac{1}{K} \sum_{k=1}^{K} V\left(F S_{k}\right)}$. One can show that

$$
\sqrt{K}\left(\frac{1}{K} \sum_{k=1}^{K} F S_{k}-E\left(\frac{1}{K} \sum_{k=1}^{K} F S_{k}\right)\right) \stackrel{d}{\longrightarrow} \mathcal{N}\left(0, \sigma_{F S}^{2}\right) .
$$

Finally,

$$
\begin{aligned}
& \sqrt{K}\left(\widehat{\Delta}-\Delta_{K}\right) \\
= & \sqrt{K}\left(\frac{\frac{1}{K} \sum_{k=1}^{K} R F_{k}}{\frac{1}{K} \sum_{k=1}^{K} F S_{k}}-\frac{E\left(\frac{1}{K} \sum_{k=1}^{K} R F_{k}\right)}{E\left(\frac{1}{K} \sum_{k=1}^{K} F S_{k}\right)}\right) \\
= & \sqrt{K} \frac{1}{E\left(\frac{1}{K} \sum_{k=1}^{K} F S_{k}\right)}\left(\frac{1}{K} \sum_{k=1}^{K} R F_{k}-E\left(\frac{1}{K} \sum_{k=1}^{K} R F_{k}\right)\right. \\
& \left.-\frac{E\left(\frac{1}{K} \sum_{k=1}^{K} R F_{k}\right)}{E\left(\frac{1}{K} \sum_{k=1}^{K} F S_{k}\right)}\left(\frac{1}{K} \sum_{k=1}^{K} F S_{k}-E\left(\frac{1}{K} \sum_{k=1}^{K} F S_{k}\right)\right)\right)+o_{P}(1) \\
= & \sqrt{K} \frac{1}{E\left(\frac{1}{K} \sum_{k=1}^{K} F S_{k}\right)}\left(\frac{1}{K} \sum_{k=1}^{K} R F_{k}-E\left(\frac{1}{K} \sum_{k=1}^{K} R F_{k}\right)-\Delta\left(\frac{1}{K} \sum_{k=1}^{K} F S_{k}-E\left(\frac{1}{K} \sum_{k=1}^{K} F S_{k}\right)\right)\right)+o_{P}(1) \\
= & \frac{F S}{E\left(\frac{1}{K} \sum_{k=1}^{K} F S_{k}\right)} \sqrt{K}\left(\frac{1}{K} \sum_{k=1}^{K}\left(\Lambda_{k}-E\left(\Lambda_{k}\right)\right)\right)+o_{P}(1) \stackrel{d}{\longrightarrow} \mathcal{N}\left(0, \sigma^{2}\right) .
\end{aligned}
$$


The first equality follows from the definitions of $F S_{k}$ and $R F_{k}$ and from (16).

The second equality follows from the fact $E\left(\frac{1}{K} \sum_{k=1}^{K} R F_{k}\right), E\left(\frac{1}{K} \sum_{k=1}^{K} F S_{k}\right), \frac{1}{K} \sum_{k=1}^{K} R F_{k}$, and $\frac{1}{K} \sum_{k=1}^{K} F S_{k}$ satisfy the assumptions of Lemma A.2. Indeed, point b) of Lemma 3.1, point c) of Assumption 4, and Assumption 3 imply that $E\left(\frac{1}{K} \sum_{k=1}^{K} F S_{k}\right) \geq \frac{3}{N^{+}}>0$. Moreover, points e.i) and e.ii) of Assumption 4 imply that $E\left(\frac{1}{K} \sum_{k=1}^{K} R F_{k}\right) / E\left(\frac{1}{K} \sum_{k=1}^{K} F S_{k}\right)$ converges towards a finite limit. Finally, it follows from (19), (20), and the fact that convergence in distribution implies boundedness in probability, that

$$
\begin{aligned}
& \sqrt{K}\left(\frac{1}{K} \sum_{k=1}^{K} R F_{k}-E\left(\frac{1}{K} \sum_{k=1}^{K} R F_{k}\right)\right)=O_{p}(1) \\
& \sqrt{K}\left(\frac{1}{K} \sum_{k=1}^{K} F S_{k}-E\left(\frac{1}{K} \sum_{k=1}^{K} F S_{k}\right)\right)=O_{p}(1) .
\end{aligned}
$$

(16), points e.i) and e.ii) of Assumption 4, and (20) ensure that

$$
\frac{1}{E\left(\frac{1}{K} \sum_{k=1}^{K} F S_{k}\right)}\left(\Delta-\frac{E\left(\frac{1}{K} \sum_{k=1}^{K} R F_{k}\right)}{E\left(\frac{1}{K} \sum_{k=1}^{K} F S_{k}\right)}\right) \sqrt{K}\left(\frac{1}{K} \sum_{k=1}^{K} F S_{k}-E\left(\frac{1}{K} \sum_{k=1}^{K} F S_{k}\right)\right)=o_{P}(1),
$$

hence the third equality. The fourth equality follows from the definition of $\Lambda_{k}$. The convergence in distribution arrow follows from a reasoning similar to that used to prove (19), and from the Slutsky lemma and the definition of FS.

Proof that $\widehat{\sigma}_{+}^{2} \stackrel{p}{\longrightarrow} \sigma_{+}^{2} \geq \sigma^{2}$.

By point a) of Assumption 1, points a) and d) of Assumption 4, the weak law of large numbers in Gut (1992), and points e.i) and e.ii) of Assumption 4,

$$
\begin{aligned}
& \frac{1}{K} \sum_{k=1}^{K} R F_{k} \stackrel{p}{\longrightarrow} \lim _{K \rightarrow+\infty} \frac{1}{K} \sum_{k=1}^{K} E\left(R F_{k}\right) \\
& \frac{1}{K} \sum_{k=1}^{K} F S_{k} \stackrel{p}{\longrightarrow} \lim _{K \rightarrow+\infty} \frac{1}{K} \sum_{k=1}^{K} E\left(F S_{k}\right) .
\end{aligned}
$$

Then, the fact that $\sqrt{K}\left(\widehat{\Delta}-\Delta_{K}\right)$ is asymptotically normal, Equation (16), and points e.i) and e.ii) of Assumption 4 imply that

$$
\widehat{\Delta} \stackrel{p}{\longrightarrow} \Delta
$$

Then, (21), (22), and the continuous mapping theorem imply that

$$
\frac{1}{K} \sum_{k=1}^{K} \widehat{\Lambda}_{k}=\frac{1}{\frac{1}{K} \sum_{k=1}^{K} F S_{k}}\left(\frac{1}{K} \sum_{k=1}^{K} R F_{k}-\widehat{\Delta} \frac{1}{K} \sum_{k=1}^{K} F S_{k}\right) \stackrel{p}{\longrightarrow} \lim _{K \rightarrow+\infty} \frac{1}{K} \sum_{k=1}^{K} E\left(\Lambda_{k}\right) .
$$


Similarly, one can show that

$$
\frac{1}{K} \sum_{k=1}^{K} \widehat{\Lambda}_{k}^{2} \stackrel{p}{\longrightarrow} \lim _{K \rightarrow+\infty} \frac{1}{K} \sum_{k=1}^{K} E\left(\Lambda_{k}^{2}\right)
$$

Then, (23), (24), and the continuous mapping theorem imply that

$$
\widehat{\sigma}_{+}^{2}=\frac{1}{K} \sum_{k=1}^{K} \widehat{\Lambda}_{k}^{2}-\left(\frac{1}{K} \sum_{k=1}^{K} \widehat{\Lambda}_{k}\right)^{2} \stackrel{p}{\longrightarrow} \sigma_{+}^{2} .
$$

Finally, the convexity of $x \mapsto x^{2}$ implies that $\frac{1}{K} \sum_{k=1}^{K} E\left(\Lambda_{k}\right)^{2} \geq\left(\frac{1}{K} \sum_{k=1}^{K} E\left(\Lambda_{k}\right)\right)^{2}$, so $\sigma_{+}^{2} \geq \sigma^{2}$. QED.

Theorem 3.2 relies on Assumption 6 below. Let

$$
\begin{aligned}
& R F_{k}^{E}=N_{k} \frac{L_{k}}{N_{k}}\left(1-\frac{L_{k}}{N_{k}}\right)\left(\frac{1}{L_{k}} \sum_{i: Z_{i k}=1} Y_{i k}-\frac{1}{N_{k}-L_{k}} \sum_{i: Z_{i k}=0} Y_{i k}\right), \\
& F S_{k}^{E}=N_{k} \frac{L_{k}}{N_{k}}\left(1-\frac{L_{k}}{N_{k}}\right) \frac{1}{L_{k}} \sum_{i: Z_{i k}=1} D_{i k} .
\end{aligned}
$$

Assumption 6 (Technical assumptions to derive the probability limit of $\widehat{\beta}_{F E}^{E}$ )

$\sup _{k \in \mathbb{N}^{*}} E\left(\left|R F_{k}^{E}\right|^{1+\delta}\right)<+\infty$ for some strictly positive $\delta ; \frac{1}{K} \sum_{k=1}^{K} E\left(\frac{S_{k}\left(N_{k}-S_{k} \frac{N_{k}+1}{T_{k}+1}\right)}{N_{k}}\right)$,

$\frac{1}{K} \sum_{k=1}^{K} E\left(\frac{S_{k}\left(N_{k}-T_{k} \frac{N_{k}+1}{T_{k}+1}\right)}{N_{k}}\left[\frac{1}{T_{k}} \sum_{i: D_{i k}(1)=1} Y_{i k}(0)-\frac{1}{N_{k}-T_{k}} \sum_{i: D_{i k}(1)=0} Y_{i k}(0)\right]\right)$, and

$\frac{1}{K} \sum_{k=1}^{K} E\left(\frac{S_{k}\left(N_{k}-S_{k} \frac{N_{k}+1}{T_{k}+1}\right)}{N_{k}} \frac{1}{T_{k}} \sum_{i: D_{i k}(1)=1}\left[Y_{i k}(1)-Y_{i k}(0)\right]\right)$ have finite limits when $K \rightarrow+\infty$.

\section{Proof of Theorem 3.2}

First,

$$
\begin{aligned}
E\left(L_{k} \mid \mathcal{P}_{k}\right) & =\sum_{l=S_{k}}^{N_{k}-T_{k}+S_{k}} l \frac{\left(\begin{array}{c}
l-1 \\
S_{k}-1
\end{array}\right)\left(\begin{array}{c}
N_{k}-l \\
T_{k}-S_{k}
\end{array}\right)}{\left(\begin{array}{l}
N_{k} \\
T_{k}
\end{array}\right)} \\
& =S_{k} \frac{N_{k}+1}{T_{k}+1} \sum_{l=S_{k}}^{N_{k}-T_{k}+S_{k}} \frac{\left(\begin{array}{c}
l \\
S_{k}
\end{array}\right)\left(\begin{array}{c}
N_{k}+1-(l+1) \\
T_{k}+1-\left(S_{k}+1\right)
\end{array}\right)}{\left(\begin{array}{l}
N_{k}+1 \\
T_{k}+1
\end{array}\right)} \\
& =S_{k} \frac{N_{k}+1}{T_{k}+1} .
\end{aligned}
$$

This derivation follows from arguments similar to those used when deriving (5). 
Then, it follows from the fact that a 2SLS coefficient with one endogenous variable and one instrument is equal to the ratio of the reduced form and first stage coefficients, from Equation (3.3.7) in Angrist \& Pischke (2008), and from the definitions of $R F_{k}^{E}$ and $F S_{k}^{E}$, that

$$
\widehat{\beta}_{F E}^{E}=\frac{\frac{1}{K} \sum_{k=1}^{K} R F_{k}^{E}}{\frac{1}{K} \sum_{k=1}^{K} F S_{k}^{E}} .
$$

For every $k$,

$$
\begin{aligned}
& E\left(R F_{k}^{E}\right) \\
& =E\left(\left(1-\frac{L_{k}}{N_{k}}\right) \sum_{i=1}^{N_{k}} Y_{i k}\left(D_{i k}(1)\right) 1\left\{R_{i k} \leq L_{k}\right\}-\frac{L_{k}}{N_{k}} \sum_{i=1}^{N_{k}} Y_{i k}(0)\left(1-1\left\{R_{i k} \leq L_{k}\right\}\right)\right) \\
& =E\left(\left(1-\frac{L_{k}}{N_{k}}\right) \sum_{i=1}^{N_{k}} Y_{i k}\left(D_{i k}(1)\right) E\left(1\left\{R_{i k} \leq L_{k}\right\} \mid L_{k}, \mathcal{P}_{k}\right)-\frac{L_{k}}{N_{k}} \sum_{i=1}^{N_{k}} Y_{i k}(0)\left(1-E\left(1\left\{R_{i k} \leq L_{k}\right\} \mid L_{k}, \mathcal{P}_{k}\right)\right)\right) \\
& =E\left(\left(1-\frac{L_{k}}{N_{k}}\right) \sum_{i=1}^{N_{k}} Y_{i k}\left(D_{i k}(1)\right)\left(D_{i k}(1) \frac{S_{k}}{T_{k}}+\left(1-D_{i k}(1)\right) \frac{L_{k}-S_{k}}{N_{k}-T_{k}}\right)\right. \\
& \left.-\frac{L_{k}}{N_{k}} \sum_{i=1}^{N_{k}} Y_{i k}(0)\left(D_{i k}(1) \frac{T_{k}-S_{k}}{T_{k}}+\left(1-D_{i k}(1)\right) \frac{N_{k}-T_{k}-L_{k}+S_{k}}{N_{k}-T_{k}}\right)\right) \\
& =E\left(\frac{\left(N_{k}-L_{k}\right) S_{k}}{N_{k}} \frac{1}{T_{k}} \sum_{i: D_{i k}(1)=1} Y_{i k}(1)-\frac{L_{k}\left(T_{k}-S_{k}\right)}{N_{k}} \frac{1}{T_{k}} \sum_{i: D_{i k}(1)=1} Y_{i k}(0)\right. \\
& \left.+\frac{\left(N_{k}-L_{k}\right)\left(L_{k}-S_{k}\right)-L_{k}\left(N_{k}-T_{k}-L_{k}+S_{k}\right)}{N_{k}} \frac{1}{N_{k}-T_{k}} \sum_{i: D_{i k}(1)=0} Y_{i k}(0)\right) \\
& =E\left(\frac{\left(N_{k}-L_{k}\right) S_{k}}{N_{k}} \frac{1}{T_{k}} \sum_{i: D_{i k}(1)=1}\left[Y_{i k}(1)-Y_{i k}(0)\right]\right. \\
& \left.+\frac{N_{k} S_{k}-L_{k} T_{k}}{N_{k}}\left(\frac{1}{T_{k}} \sum_{i: D_{i k}(1)=1} Y_{i k}(0)-\frac{1}{N_{k}-T_{k}} \sum_{i: D_{i k}(1)=0} Y_{i k}(0)\right)\right) \\
& =E\left(\frac{S_{k}\left(N_{k}-S_{k} \frac{N_{k}+1}{T_{k}+1}\right)}{N_{k}} \frac{1}{T_{k}} \sum_{i: D_{i k}(1)=1}\left[Y_{i k}(1)-Y_{i k}(0)\right]\right. \\
& \left.+\frac{S_{k}\left(N_{k}-T_{k} \frac{N_{k}+1}{T_{k}+1}\right)}{N_{k}}\left(\frac{1}{T_{k}} \sum_{i: D_{i k}(1)=1} Y_{i k}(0)-\frac{1}{N_{k}-T_{k}} \sum_{i: D_{i k}(1)=0} Y_{i k}(0)\right)\right) .
\end{aligned}
$$

The first equality follows from the definition of $R F_{k}^{E}$ and some algebra. The second equality follows from the law of iterated expectations and the linearity of the conditional expectation. The third equality follows from (7) and (8). The fourth and fifth equality follow from some algebra. The last equality follows from the law of iterated expectations, the linearity of the conditional expectation, and (26). 
Similarly, one can show that for every $k$,

$$
E\left(F S_{k}^{E}\right)=E\left(\frac{S_{k}\left(N_{k}-S_{k} \frac{N_{k}+1}{T_{k}+1}\right)}{N_{k}}\right) .
$$

Equations (28) and (29) combined with Assumption 6 imply that $\frac{1}{K} \sum_{k=1}^{K} E\left(R F_{k}^{E}\right)$ and $\frac{1}{K} \sum_{k=1}^{K} E\left(F S_{k}^{E}\right)$ converge towards finite limits when $K \rightarrow+\infty$. Then, one can use a reasoning similar to that used to prove (21) to show that

$$
\widehat{\beta}_{F E}^{E} \stackrel{p}{\longrightarrow} \frac{\lim _{K \rightarrow+\infty} \frac{1}{K} \sum_{k=1}^{K} E\left(R F_{k}^{E}\right)}{\lim _{K \rightarrow+\infty} \frac{1}{K} \sum_{k=1}^{K} E\left(F S_{k}^{E}\right)} .
$$

The result follows from plugging (28) and (29) into (30). QED.

\section{Proof of Theorem 3.3}

\section{Proof of a)}

The organization of the proof is as follows. We use the variance decomposition formula

$$
K V(\widehat{\Delta})=E\left(K V\left(\widehat{\Delta} \mid\left(\mathcal{D}_{k}, \mathcal{R}_{k}\right)_{1 \leq k \leq K}\right)\right)+K V\left(E\left(\widehat{\Delta} \mid\left(\mathcal{D}_{k}, \mathcal{R}_{k}\right)_{1 \leq k \leq K}\right)\right),
$$

and then compute the limsup of the first term on the right hand side, before showing that the second term is equal to 0 .

Let $\widehat{F S}=\frac{1}{K} \sum_{k=1}^{K} \frac{N_{k}}{\bar{N}} \frac{S_{k}-1}{L_{k}-1}$.

$$
\begin{aligned}
& K V\left(\widehat{\Delta} \mid\left(\mathcal{D}_{k}, \mathcal{R}_{k}\right)_{1 \leq k \leq K}\right) \\
= & \frac{K}{\widehat{F S}^{2}} V\left(\frac{1}{K} \sum_{k=1}^{K} \frac{N_{k}}{\bar{N}}\left(\frac{1}{L_{k}-1} \sum_{i: Z_{i k}=1} w_{i k}\left(Y_{i k}(0)+\tau D_{i k}(1)\right)-\frac{1}{N_{k}-L_{k}} \sum_{i: Z_{i k}=0} Y_{i k}(0)\right) \mid\left(\mathcal{D}_{k}, \mathcal{R}_{k}\right)_{1 \leq k \leq K}\right) \\
= & \frac{K}{\widehat{F S}^{2}} V\left(\frac{1}{K} \sum_{k=1}^{K} \frac{N_{k}}{\bar{N}}\left(\frac{1}{L_{k}-1} \sum_{i: Z_{i k}=1} w_{i k} Y_{i k}(0)-\frac{1}{N_{k}-L_{k}} \sum_{i: Z_{i k}=0} Y_{i k}(0)\right) \mid\left(\mathcal{D}_{k}, \mathcal{R}_{k}\right)_{1 \leq k \leq K}\right) \\
= & \frac{1}{\widehat{F S}^{2}} \frac{1}{K} \sum_{k=1}^{K} \frac{N_{k}^{2}}{\bar{N}^{2}}\left(\frac{1}{\left(L_{k}-1\right)^{2}} \sum_{i: Z_{i k}=1} w_{i k}^{2} V\left(Y_{i k}(0) \mid\left(\mathcal{D}_{k}, \mathcal{R}_{k}\right)_{1 \leq k \leq K}\right)\right. \\
& +\frac{1}{\left.N_{k}-L_{k}\right)^{2}} \sum_{i: Z_{i k}=0}^{\left.V\left(Y_{i k}(0) \mid\left(\mathcal{D}_{k}, \mathcal{R}_{k}\right)_{1 \leq k \leq K}\right)\right)} \\
= & \frac{\sigma_{Y(0)}^{2}}{\widehat{F S}^{2}} \frac{1}{K} \sum_{k=1}^{K} \frac{N_{k}^{2}}{\bar{N}^{2}}\left(\frac{1}{\left(L_{k}-1\right)^{2}} \sum_{i: Z_{i k}=1} w_{i k}^{2}+\frac{1}{N_{k}-L_{k}}\right) \\
\leq & \frac{\sigma_{Y(0)}^{2}}{\widehat{F S}^{2}} \frac{1}{K} \sum_{k=1}^{K} \frac{N_{k}^{2}}{\bar{N}^{2}}\left(\frac{1}{L_{k}-1}+\frac{1}{N_{k}-L_{k}}\right) .
\end{aligned}
$$


The first equality follows from the fact that $\widehat{F S}$ is a function of $\left(\mathcal{D}_{k}, \mathcal{R}_{k}\right)_{1 \leq k \leq K}$, and from point d) of Assumption 5. The second equality follows from the fact that conditional on $\left(\mathcal{D}_{k}, \mathcal{R}_{k}\right)_{1 \leq k \leq K}, \frac{1}{K} \sum_{k=1}^{K} \frac{N_{k}}{N} \frac{1}{L_{k}-1} \sum_{i: Z_{i k}=1} w_{i k} D_{i k}(1) \tau$ is a constant. The third equality follows from point a) of Assumption 5. The fourth equality follows from point b) of Assumption 5. The inequality follows from the fact that $0 \leq w_{i k} \leq 1$ implies $0 \leq w_{i k}^{2} \leq w_{i k}$, and $\sum_{i: Z_{i k}=1} w_{i k}=L_{k}-1$.

Assumption 3 and point c) of Assumption 4 guarantee that for all $k$,

$$
\frac{N_{k}^{2}}{\bar{N}^{2}}\left(\frac{1}{L_{k}-1}+\frac{1}{N_{k}-L_{k}}\right) \leq 2\left(\frac{N^{+}}{3}\right)^{2} .
$$

Moreover, by point a) of Assumption 4 these random variables are independent, so it follows from Gut (1992) that

$$
\frac{1}{K} \sum_{k=1}^{K} \frac{N_{k}^{2}}{N^{2}}\left(\frac{1}{L_{k}-1}+\frac{1}{N_{k}-L_{k}}-E\left(\frac{1}{L_{k}-1}+\frac{1}{N_{k}-L_{k}}\right)\right) \stackrel{p}{\longrightarrow} 0 .
$$

Using a reasoning similar to that used in the proof of Lemma A.1, one can show that

$$
E\left(\frac{1}{L_{k}-1}+\frac{1}{N_{k}-L_{k}}\right)=\frac{T_{k}}{N_{k}}\left(\frac{1}{S_{k}-1}+\frac{1}{T_{k}-S_{k}}\right)=\frac{T_{0}}{N_{0}}\left(\frac{1}{S_{0}-1}+\frac{1}{T_{0}-S_{0}}\right),
$$

where the second equality follows from point e) of Assumption 5. Combining the two preceding displays,

$$
\frac{1}{K} \sum_{k=1}^{K} \frac{N_{k}^{2}}{\bar{N}^{2}}\left(\frac{1}{L_{k}-1}+\frac{1}{N_{k}-L_{k}}\right) \stackrel{p}{\longrightarrow} \frac{T_{0}}{N_{0}}\left(\frac{1}{S_{0}-1}+\frac{1}{T_{0}-S_{0}}\right) .
$$

Similarly, one can show that

$$
\frac{1}{K} \sum_{k=1}^{K} \frac{N_{k}}{\bar{N}} \frac{S_{k}-1}{L_{k}-1} \stackrel{p}{\longrightarrow} \frac{T_{0}}{N_{0}} .
$$

Then, it follows from (34), (35), and the continuous mapping theorem that

$$
\frac{\sigma_{Y(0)}^{2}}{\widehat{F S}^{2}} \frac{1}{K} \sum_{k=1}^{K} \frac{N_{k}^{2}}{\bar{N}^{2}}\left(\frac{1}{L_{k}-1}+\frac{1}{N_{k}-L_{k}}\right) \stackrel{p}{\longrightarrow} \sigma_{Y(0)}^{2} \frac{\frac{1}{S_{0}-1}+\frac{1}{T_{0}-S_{0}}}{\frac{T_{0}}{N_{0}}} .
$$

Finally, Assumption 3 and point c) of Assumption 4 imply that $\widehat{F S} \geq \frac{1}{N^{+}-2}$. Combined with (33), this implies that $\frac{\sigma_{Y(0)}^{2}}{\widehat{F S}^{2}} \frac{1}{K} \sum_{k=1}^{K} \frac{N_{k}^{2}}{\bar{N}^{2}}\left(\frac{1}{L_{k}-1}+\frac{1}{N_{k}-L_{k}}\right)$ is bounded. Then, it follows from Equation (36) and Theorem 2.20 in Van der Vaart (2000) that

$$
\lim _{K \rightarrow+\infty} E\left(\frac{\sigma_{Y(0)}^{2}}{\widehat{F S}^{2}} \frac{1}{K} \sum_{k=1}^{K} \frac{N_{k}^{2}}{\bar{N}^{2}}\left(\frac{1}{L_{k}-1}+\frac{1}{N_{k}-L_{k}}\right)\right)=\sigma_{Y(0)}^{2} \frac{\frac{1}{S_{0}-1}+\frac{1}{T_{0}-S_{0}}}{\frac{T_{0}}{N_{0}}} .
$$

Combined with Equation (32), Equation (37) implies that

$$
\limsup _{K \rightarrow+\infty} E\left(K V\left(\widehat{\Delta} \mid\left(\mathcal{D}_{k}, \mathcal{R}_{k}\right)_{1 \leq k \leq K}\right)\right) \leq \sigma_{Y(0)}^{2} \frac{\frac{1}{S_{0}-1}+\frac{1}{T_{0}-S_{0}}}{\frac{T_{0}}{N_{0}}} .
$$


Then,

$$
\begin{aligned}
& V\left(E\left(\widehat{\Delta} \mid\left(\mathcal{D}_{k}, \mathcal{R}_{k}\right)_{1 \leq k \leq K}\right)\right) \\
= & V\left(\frac{1}{\widehat{F S}} E\left(\frac{1}{K} \sum_{k=1}^{K} \frac{N_{k}}{\bar{N}}\left(\frac{1}{L_{k}-1} \sum_{i: Z_{i k}=1} w_{i k}\left(Y_{i k}(0)+\tau D_{i k}(1)\right)-\frac{1}{N_{k}-L_{k}} \sum_{i: Z_{i k}=0} Y_{i k}(0)\right) \mid\left(\mathcal{D}_{k}, \mathcal{R}_{k}\right)_{1 \leq k \leq K}\right)\right) \\
= & V\left(\tau+\frac{1}{\widehat{F S}} \frac{1}{K} \sum_{k=1}^{K} \frac{N_{k}}{\bar{N}}\left(\frac{1}{L_{k}-1} \sum_{i: Z_{i k}=1} w_{i k} E\left(Y_{i k}(0) \mid\left(\mathcal{D}_{k}, \mathcal{R}_{k}\right)_{1 \leq k \leq K}\right)\right.\right. \\
& \left.\left.-\frac{1}{N_{k}-L_{k}} \sum_{i: Z_{i k}=0} E\left(Y_{i k}(0) \mid\left(\mathcal{D}_{k}, \mathcal{R}_{k}\right)_{1 \leq k \leq K}\right)\right)\right) \\
= & V\left(\frac{1}{\widehat{F S}} \frac{1}{K} \sum_{k=1}^{K} \frac{N_{k}}{\bar{N}} \mu_{k}\left(\frac{1}{L_{k}-1} \sum_{i: Z_{i k}=1} w_{i k}-1\right)\right) \\
= & 0 .
\end{aligned}
$$

The first equality follows from the fact that $\widehat{F S}$ is a function of $\left(\mathcal{D}_{k}, \mathcal{R}_{k}\right)_{1 \leq k \leq K}$, and from point d) of Assumption 5. The second equality follows from $\frac{1}{K} \sum_{k=1}^{K} \frac{N_{k}}{\bar{N}} \frac{1}{L_{k}-1} \sum_{i: Z_{i k}=1} w_{i k} D_{i k}(1)=$ $\widehat{F S}$. The third equality follows from point c) of Assumption 5. The last equality follows from $\sum_{i: Z_{i k}=1} w_{i k}=L_{k}-1$.

Finally, the result follows from Equations (31), (38) and (39).

\section{Proof of $b$ )}

The proof if fairly similar to that of Point a), so we just sketch it.

Let $\widehat{F S}_{I}=\frac{1}{K} \sum_{k=1}^{K}\left(\frac{\sum_{i: Z_{i k}^{\prime}=1} D_{i k}(1)}{S_{0}}-\frac{S_{0}-\sum_{i: Z_{i k}^{\prime}=1} D_{i k}(1)}{N_{0}-S_{0}}\right)$. One can show that under points a), b), d), and e) of Assumption 5,

$$
K V\left(\widehat{\beta}_{P S}^{I} \mid\left(\mathcal{D}_{k}, \mathcal{R}_{k}\right)_{1 \leq k \leq K}\right)=\frac{\sigma_{Y(0)}^{2}\left(\frac{1}{S_{0}}+\frac{1}{N_{0}-S_{0}}\right)}{\widehat{F S}_{I}^{2}} .
$$

Then, for every $k$

$$
E\left(\sum_{i: Z_{i k}^{\prime}=1} D_{i k}(1)\right)=E\left(\sum_{i=1}^{N_{0}} 1\left\{R_{i k} \leq S_{0}\right\} D_{i k}(1)\right)=E\left(\sum_{i=1}^{N_{0}} D_{i k}(1) \frac{S_{0}}{N_{0}}\right)=\frac{S_{0} T_{0}}{N_{0}} .
$$

The second equality follows from the law of iterated expectations, Assumption 2, and from point e) of Assumption 5. The third follows from point e) of Assumption 5.

It follows from the previous display that for every $k$,

$$
E\left(\frac{\sum_{i: Z_{i k}^{\prime}=1} D_{i k}(1)}{S_{0}}-\frac{S_{0}-\sum_{i: Z_{i k}^{\prime}=1} D_{i k}(1)}{N_{0}-S_{0}}\right)=\frac{T_{0}}{N_{0}}-\frac{S_{0}-S_{0} \frac{T_{0}}{N_{0}}}{N_{0}-S_{0}}=\frac{T_{0}-S_{0}}{N_{0}-S_{0}} .
$$


Combining Equations (40) and (41), one can then show that

$$
K V\left(\widehat{\beta}_{P S}^{I} \mid\left(\mathcal{D}_{k}, \mathcal{R}_{k}\right)_{1 \leq k \leq K}\right) \stackrel{p}{\longrightarrow} \frac{\sigma_{Y(0)}^{2}\left(\frac{1}{S_{0}}+\frac{1}{N_{0}-S_{0}}\right)}{\left(\frac{T_{0}-S_{0}}{N_{0}-S_{0}}\right)^{2}}
$$

Finally, $\widehat{F S}_{I}>\frac{S_{0}-\left(N_{0}-T_{0}\right)}{S_{0}}-\frac{N_{0}-T_{0}}{N_{0}-S_{0}}$, which is stricly positive under point f) of Assumption 5 . Together with Equation (40) and Assumption 3, this implies that $K V\left(\widehat{\beta}_{P S}^{I} \mid\left(\mathcal{D}_{k}, \mathcal{R}_{k}\right)_{1 \leq k \leq K}\right)$ is bounded, so Equation (42) implies that

$$
\lim _{K \rightarrow+\infty} E\left(K V\left(\widehat{\beta}_{P S}^{I} \mid\left(\mathcal{D}_{k}, \mathcal{R}_{k}\right)_{1 \leq k \leq K}\right)\right)=\frac{\sigma_{Y(0)}^{2}\left(\frac{1}{S_{0}}+\frac{1}{N_{0}-S_{0}}\right)}{\left(\frac{T_{0}-S_{0}}{N_{0}-S_{0}}\right)^{2}} .
$$

Then, one can follow the steps used to prove Equation (39) to show that

$$
V\left(E\left(\widehat{\beta}_{P S}^{I} \mid\left(\mathcal{D}_{k}, \mathcal{R}_{k}\right)_{1 \leq k \leq K}\right)\right)=0
$$

Finally, the result follows from the variance decomposition formula and Equations (43) and (44).

Proof of the sufficient condition for $\limsup _{K \rightarrow+\infty} V\left(\sqrt{K}\left(\widehat{\Delta}-\Delta_{K}\right)\right) \leq \lim _{K \rightarrow+\infty} V\left(\sqrt{K}\left(\widehat{\beta}_{P S}^{I}-\Delta_{K}\right)\right)$

One has $0<\frac{T_{0}-S_{0}}{N_{0}-S_{0}} \leq \frac{T_{0}}{N_{0}}$, so

$$
\frac{\frac{1}{S_{0}-1}+\frac{1}{T_{0}-S_{0}}}{\frac{T_{0}}{N_{0}}} \leq \frac{\frac{1}{S_{0}-1}+\frac{1}{T_{0}-S_{0}}}{\frac{T_{0}-S_{0}}{N_{0}-S_{0}}}
$$

Then,

$$
\frac{\frac{1}{S_{0}-1}+\frac{1}{T_{0}-S_{0}}}{\frac{T_{0}-S_{0}}{N_{0}-S_{0}}} \leq \frac{\frac{1}{S_{0}}+\frac{1}{N_{0}-S_{0}}}{\left(\frac{T_{0}-S_{0}}{N_{0}-S_{0}}\right)^{2}} \Leftrightarrow \frac{T_{0}-S_{0}}{N_{0}-S_{0}} \frac{1}{S_{0}-1}+\frac{1}{N_{0}-S_{0}} \leq \frac{1}{S_{0}}+\frac{1}{N_{0}-S_{0}} \Leftrightarrow \frac{T_{0}-S_{0}}{N_{0}-S_{0}} \leq 1-\frac{1}{S_{0}} .
$$

The result follows from the two preceding displays, and using again the fact that $\frac{T_{0}-S_{0}}{N_{0}-S_{0}} \leq \frac{T_{0}}{N_{0}}$. 